\title{
On the Bartlett correction of empirical likelihood for Gaussian long-memory time series
}

\author{
Ngai Hang Chan ${ }^{1,2}$, Kun Chen ${ }^{2}$, Chun Yip Yau ${ }^{1}$ \\ ${ }^{1}$ Department of Statistics \\ Chinese University of Hong Kong \\ Shatin, N.T. \\ Hong Kong \\ e-mail: nhchan@sta.cuhk.edu.hk; cyyau@sta.cuhk.edu.hk \\ ${ }^{2}$ School of Statistics \\ Southwestern University of Finance and Economics \\ Chengdu \\ China \\ e-mail: chenkunmath@gmail.com
}

\begin{abstract}
Bartlett correction is one of the desirable features of empirical likelihood (EL) since it allows constructions of confidence regions with improved coverage probabilities. Previous studies demonstrated the Bartlett correction of EL for independent observations and for short-memory time series. By establishing the validity of Edgeworth expansion for the signed root empirical log-likelihood ratio, the validity of Bartlett correction of EL for Gaussian long-memory time series is established. In particular, orders of the coverage error of confidence regions can be reduced from $\log ^{6} n / n$ to $\log ^{3} n / n$, which is different from the classical rate of reduction from $n^{-1}$ to $n^{-2}$.
\end{abstract}

AMS 2000 subject classifications: 62F10, 62M10.

Keywords and phrases: Coverage error, Edgeworth expansion, periodogram, Whittle likelihood.

Received May 2014.

\section{Introduction}

Empirical likelihood (EL) is a nonparametric likelihood approach of statistical inference introduced by Owen $[30,31,32]$. Let $X_{1}, X_{2}, \ldots, X_{n}$ be a sequence of independent and identically distributed (i.i.d.) random variables satisfying $\mathrm{E}\left(m\left(X_{j}, \theta\right)\right)=0$, where $\theta \in \mathbb{R}^{k}$ and $m\left(X_{j}, \theta\right) \in \mathbb{R}^{k}$ is a vector-valued estimating equation. The sample may be regarded as coming from a discrete probability distribution concentrated only at the values $m\left(X_{j}, \theta\right)$. The EL is the largest probability to attain the observed sample,

$$
\operatorname{EL}(\theta)=\max _{p_{j}}\left\{\prod_{j=1}^{n} p_{j} \mid \sum_{j=1}^{n} p_{j} m\left(X_{j}, \theta\right)=0, \sum_{j=1}^{n} p_{j}=1, p_{j} \geq 0\right\} .
$$


It can be shown that $\operatorname{EL}(\theta)=n^{-n}$ without the constraint $\sum_{j=1}^{n} p_{j} m\left(X_{j}, \theta\right)=0$. Thus the profile EL ratio for the parameter of interest $\theta$ is attained through dividing $\operatorname{EL}(\theta)$ by $n^{-n}$, i.e., $\mathcal{R}(\theta)=\operatorname{EL}(\theta) / n^{-n} \in[0,1]$. By the method of Lagrange multiplier, $\mathcal{R}(\theta)=\prod_{j=1}^{n} n \hat{p}_{j}$, where $\hat{p}_{j}=1 / n\left(1+t^{T} m\left(X_{j}, \theta\right)\right)$, and $t$ is the solution of equation

$$
\frac{1}{n} \sum_{j=1}^{n} \frac{m\left(X_{j}, \theta\right)}{1+t^{T} m\left(X_{j}, \theta\right)}=0 .
$$

Therefore, the logarithm of the profile EL ratio is

$$
l(\theta)=-2 \log \mathcal{R}(\theta)=2 \sum_{j=1}^{n} \log \left(1+t^{T} m\left(X_{j}, \theta\right)\right) .
$$

Qin and Lawless [34] proved that for just-identified estimating equations (i.e. the number of estimating equations is the same as that of parameters) and true parameter $\theta_{0}$,

$$
l\left(\theta_{0}\right) \stackrel{d}{\rightarrow} \chi_{k}^{2} \quad \text { as } n \rightarrow \infty,
$$

which resembles the Wilks's theorem for parametric likelihood. Consequently, an asymptotic $100(1-\alpha) \%$ EL confidence region for $\theta_{0}$ can be constructed by $\mathbf{I}_{\alpha}=\left\{\theta \mid l(\theta) \leq \chi_{k, 1-\alpha}^{2}\right\}$, where $\chi_{k, 1-\alpha}^{2}$ is $1-\alpha$ quantile of chi-squared distribution with $k$ degrees of freedom.

The standard theory of EL requires the estimating functions $\left\{m\left(X_{j}, \theta\right)\right\}$ to be independent across $j$. Monti [27] extended EL to time series models by considering

$$
m_{j}(\theta)=\frac{\partial \log \left\{f\left(\omega_{j}, \theta\right)\right\}}{\partial \theta}\left\{\frac{I_{n}\left(\omega_{j}\right)}{f\left(\omega_{j}, \theta\right)}-1\right\},
$$

which is the score function of Whittle likelihood

$$
W L(\theta)=-\sum_{j=1}^{n} \log \left\{f\left(\omega_{j}, \theta\right)\right\}-\sum_{j=1}^{n} \frac{I_{n}\left(\omega_{j}\right)}{f\left(\omega_{j}, \theta\right)}
$$

where $I_{n}\left(\omega_{j}\right)=\frac{1}{2 \pi n}\left|\sum_{t=1}^{n} X_{t} \exp \left(-i \omega_{j} t\right)\right|^{2},(i=\sqrt{-1})$, is the periodogram ordinate at Fourier frequency $\omega_{j}=2 \pi j / n$ for $j \in \mathbb{S}_{n}=\{1, \ldots, n\}$ and $f\left(\omega_{j}, \theta\right)$ is the spectral density function, belonging to a class of parametric family. Since the periodogram ordinates are asymptotically independent for Gaussian shortmemory time series [6], the $m_{j}(\theta)$ in (1.3) are asymptotically independent estimating functions and the Wilks's theorem for the periodogram-based EL statistic can be established. Treating the periodogram collections $\left\{I_{n}\left(\omega_{j}\right): j \in \mathbb{S}_{n}\right\}$ as approximate independent in bootstrap context is discussed in Hurvich and Zeger [23], and Kreiss and Paparoditis [25]. However, the dependence among the periodogram collection creates problems in estimation the dependence structure when applied to Whittle score function [14]. Specifically, the dependence between different ordinates for non-Gaussian processes leads to an extra term, which will vanish for Gaussian processes, in the asymptotic variance of Whittle 
score function. Based on a different Whittle-type estimating function, Ogata [29] considered EL for non-Gaussian stationary processes. This frequency domain EL method is extended to a general framework included both short- and long-range dependent series by Nordman and Lahiri [28].

Meanwhile, other approaches to EL in time series are considered. Kitamura [24] studied blockwise EL for weakly dependent series. Chuang and Chan [12] used martingale estimating equation to unstable autoregressive models. Chan and Ling [8] extended this methodology to GARCH models.

One attractive feature of EL is Bartlett correction, which is a simple adjustment to the log-EL ratio that improves the approximation to the limiting chi-squared distribution and hence achieves a better coverage accuracy. Define the coverage error of EL confidence region by

$$
E_{\alpha}=P\left(l\left(\theta_{0}\right) \leq \chi_{k, 1-\alpha}^{2}\right)-(1-\alpha) .
$$

Zhang [38] showed that for $k=1, E_{\alpha}$ converges to 0 as $n \rightarrow \infty$ with order $O\left(n^{-1}\right)$, which can be reduced to $O\left(n^{-2}\right)$ by Bartlett correction technique. Hall and La Scala [19] demonstrated the Bartlett correction for EL in the case $\theta=\mu$, the population mean. Bartlett correction for smooth functions of means is established by DiCiccio, Hall and Romano [15]. Chen and Cui [10] studied Bartlett correction of EL for over-identified models (i.e., the number of estimating functions is larger than that of parameters) in econometrics. Chan and Liu [9], based on the periodogram version of EL, showed that $E_{\alpha}$ can be reduced from order $O\left(n^{-1}\right)$ to $o\left(n^{-1}\right)$ for Gaussian short-memory time series.

It is unclear, however, whether Bartlett correction is applicable in longmemory time series (LMTS). Hurvich and Beltrao [22] and Robinson [35] proved that the periodogram ordinates of LMTS are asymptotically dependent for frequencies near the origin. Therefore, the proof of Bartlett correction of EL for weakly dependent data, which relies on the asymptotic independence of periodograms, cannot be directly generalized to LMTS. In this paper, by establishing the validity of the Edgeworth expansion for Gaussian LMTS, we prove that EL is moderately Bartlett correctable, in the sense that, $E_{\alpha}$ is reduced from order $\log ^{6} n / n$ to $\log ^{3} n / n$. Although we only establish the Bartlett correctability of EL for Gaussian distributed time series, this exploration provides a fundamental step for further research in non-Gaussian cases. Moreover, our simulation results demonstrate that the performance of Bartlett corrected EL is better than that of the Bartlett corrected version of Whittle likelihood in ARFIMA models with Gaussian noise, which justifies the usefulness of Bartlett correction of EL for Gaussian distributed series.

This paper is organized as follows. Section 2 reviews LMTS models and Bartlett correction of EL for i.i.d. observations and short-memory time series. In Section 3, we establish the validity of Edgeworth expansion, which provides a fundamental tool for the main results. Finally, Section 4 presents simulation studies that demonstrate the good finite sample performance of Bartlett correction in ARFIMA models. Furthermore, we study the coverage error of both Whittle likelihood ratio and Bartlett corrected statistics. Proofs of technical results are given in the Appendix. 
Throughout the paper, the following notations will be adopted: $O(1)\left(O_{p}(1)\right)$ means a term (a random variable) which is bounded (in probability); $o(1)\left(o_{p}(1)\right)$ denotes a term (a random variable) converging to zero (in probability); " $a \sim b$ " means $a / b \rightarrow 1 ; \mathbb{R}^{k}$ denotes the Euclidean space with dimension $k$; and $K$ is a generic constant.

\section{Review of long-memory time series and Bartlett correction}

\subsection{Long-memory time series}

Consider a weakly stationary real-valued process $\left\{X_{t}\right\}$ with mean zero and spectral density function

$$
f(\omega, \theta) \sim K \omega^{-\theta} \quad \text { as } \omega \rightarrow 0^{+},
$$

where $K>0$ and $\theta \in \mathbb{R}$. The parameter $\theta$ is known as the memory parameter. The process $X_{t}$ is said to have short-memory when $\theta=0$, long-memory when $\theta \in$ $(0,1)$, and negative-memory when $\theta \in(-1,0)$. This model includes two widely used long-memory parametric models: the ARFIMA model $[17,20]$, where the fractional parameter $d$ is defined by $d=\theta / 2$, and the fractional Gaussian noise model [26], where the self-similar parameter $H$ satisfies $H=(\theta+1) / 2$. For details, see Beran [4].

Note that the spectral density function is unbounded but integrable at the origin. Due to this singularity, periodogram ordinate is asymptotically biased estimators for spectral density at low frequencies (see Lemma B.4 below). Different periodogram ordinates are also asymptotically correlated when the frequencies tend to zero (see Lemma B.4 below). However, when associated Fourier frequencies are separated by a fixed distance, periodograms are asymptotically uncorrelated (see Lemmas B.2 and B.3 below).

\subsection{Bartlett correction of $\mathrm{EL}$}

For i.i.d. real-valued estimating functions, it can be shown that $\mathrm{E}\left(l\left(\theta_{0}\right)\right)=1+$ $b / n+O\left(n^{-2}\right)$, for some constant $b$. Bartlett correction corresponds to multiplying a scaling factor $1+b n^{-1}$ to the pivot $\chi_{1,1-\alpha}^{2}$. Then the Bartlett corrected EL confidence interval is given by $\mathbf{I}_{\alpha}^{\prime}=\left\{\theta \mid l(\theta) \leq\left(1+\frac{b}{n}\right) \chi_{1,1-\alpha}^{2}\right\}$. Define the signed root empirical log-likelihood ratio $S R=R_{1}+R_{2}+R_{3}$ by

$$
l\left(\theta_{0}\right)=n S R^{2}+R_{n}=n\left(R_{1}+R_{2}+R_{3}\right)^{2}+R_{n},
$$

where $R_{1}=O_{p}\left(n^{-1 / 2}\right), R_{2}=O_{p}\left(n^{-1}\right), R_{3}=O_{p}\left(n^{-3 / 2}\right)$ and $R_{n}=O_{p}\left(n^{-3 / 2}\right)$. Bartlett correction of EL bases on the Edgeworth expansion for the probability density function (p.d.f.) of $\sqrt{n} S R$. The idea behind this is to prove that the distribution of squared root of EL converges to normal. For example, in the i.i.d. case, the density admits the formula expansion

$$
\pi(x)=\phi(x)+n^{-1 / 2} r_{1}(x) \phi(x)+n^{-1} r_{2}(x) \phi(x)+n^{-3 / 2} r_{3}(x) \phi(x)+O\left(n^{-2}\right),
$$


where $\phi(x)$ is the p.d.f. of a standard normal random variable; $r_{1}$ and $r_{3}$ are odd polynomials and $r_{2}$ is an even polynomial of degree 2 . Unlike other statistics in general, $r_{2}$ does not involve terms of degree 4 and 6 . This feature makes EL Bartlett correctable valid because the scaling of EL completely removes all terms of order $n^{-1}$ in (2.2). Letting $c_{\alpha}=\chi_{1,1-\alpha}^{2}$ and $c_{\alpha}^{\prime}=c_{\alpha}\left(1+b n^{-1}\right)$, we have

$$
\begin{aligned}
P\left(\theta_{0} \in \mathbf{I}_{\alpha}^{\prime}\right)= & P\left(l\left(\theta_{0}\right) \leq c_{\alpha}^{\prime}\right)=P\left((\sqrt{n} S R)^{2}+R_{n} \leq c_{\alpha}^{\prime}\right) \\
= & \int_{-\sqrt{c_{\alpha}^{\prime}}}^{\sqrt{c_{\alpha}^{\prime}}} \phi(x) d x+\int_{-\sqrt{c_{\alpha}^{\prime}}}^{\sqrt{c_{\alpha}^{\prime}}}\left\{n^{-1 / 2} r_{1}(x) \phi(x)+n^{-1} r_{2}(x) \phi(x)\right. \\
& \left.+n^{-3 / 2} r_{3}(x) \phi(x)\right\} d x+O\left(n^{-3 / 2}\right)=1-\alpha+O\left(n^{-3 / 2}\right),
\end{aligned}
$$

where the $O\left(n^{-3 / 2}\right)$ in the last equality is in fact $O\left(n^{-2}\right)$ [3]. Thus Bartlett correction improves the coverage accuracy from $O\left(n^{-1}\right)$ to $O\left(n^{-2}\right)$. For shortmemory time series, we have

$$
\mathrm{E}\left(I_{n}\left(\omega_{j}\right)\right)=f\left(\omega_{j}, \theta_{0}\right)-\frac{c}{n}+o\left(n^{-2}\right)
$$

where

$$
c=\frac{1}{2 \pi} \sum_{j=-\infty}^{\infty}|j| \gamma(j) e^{-i j \omega_{j}}
$$

It can be seen from (2.3) that periodograms are asymptotic unbiased estimators for spectral density functions, with convergence rate $O\left(n^{-1}\right)$. In this case, Chan and Liu [9] constructed the profile EL estimating function through $m_{j}(\theta)$ as Monti [27]. Through deriving orders of first four moments of $m_{j}(\theta)$, they obtained the coefficients of polynomials in Edgeworth expansion (2.2). In particular, the expectation of the sample mean function at the true value $\theta_{0}$ is

$$
\mathrm{E}(\bar{m})=\mathrm{E}\left[\frac{1}{n} \sum_{j=1}^{n} m_{j}\left(\theta_{0}\right)\right] \sim K n^{-1}
$$

where $m_{j}\left(\theta_{0}\right)$ is given by (1.3). Because of the order $n^{-1}$ bias, terms of degrees 4 and 6 in $r_{2}$, terms of degree 3 in $r_{1}$ and degree 5 in $r_{3}$ vanish. Based on these results, they deduced the Edgeworth expansion of signed root empirical loglikelihood with a different form for Gaussian short-memory time series, leading to an inaccurate improvement of coverage accuracy. For LMTS, however, (2.3) does not hold, i.e., $\mathrm{E}(\bar{m})$ does not converge to zero at rate $n^{-1}$. In Lemma 3.1 below, we obtain the order $O\left(\log ^{3} n / n\right)$ of $\mathrm{E}(\bar{m})$ in LMTS by carefully bounding the summation in $\bar{m}$ over different ranges of frequencies in the entire collection. Due to this larger bias involving $\log n$, an irregular form Edgeworth expansion is established to show a "slight" Bartlett correctability in the next Section. 


\section{Main results}

\subsection{Assumptions}

Before establishing the validity of Edgeworth expansion and Bartlett correction, we impose the following Assumptions.

\section{Assumptions.}

1. $\left\{X_{t}\right\}$ is a real-valued linear weakly stationary process satisfying

$$
X_{t}=\sum_{u=0}^{\infty} a_{u} \epsilon_{t-u}
$$

where $a_{0}=1$, and $\sum_{u=0}^{\infty} a_{u}^{2}<\infty$. The noise process $\left\{\epsilon_{t}\right\}$ is a sequence of Gaussian i.i.d. random variables with $\mathrm{E}\left(\epsilon_{t}\right)=0$ and finite known innovation variance $\mathrm{E}\left(\epsilon_{t}^{2}\right)=\sigma_{\epsilon}^{2}$.

2. The spectral density function of $\left\{X_{t}\right\}$ is given by

$$
f(\omega, \theta)=\frac{\sigma_{\epsilon}^{2}}{\omega^{2 d}} f^{*}(\omega)
$$

where $d=\theta / 2 \in\left(0, \frac{1}{2}\right)$ is parameter of interest, and $f^{*}(\omega)$ is an even, positive, continuous function on $[-\pi, \pi]$, bounded above and away from zero. In addition, we assume that the true spectral density $(\sqrt{-1}=i)$,

$$
f(\omega)=\frac{1}{2 \pi} \sum_{k=-\infty}^{\infty} \operatorname{Cov}\left(X_{t}, X_{t+k}\right) \exp (-i \omega k)=f(\omega, \theta) .
$$

The Gaussian assumption is used to deduce bounds for higher order moments of $\bar{m}$ (see Lemma B.7 below). This condition rules out distributions supported on lattice, and implies Cramér's condition $\lim \sup _{\tau \rightarrow \infty}\left|\mathrm{E}\left(\exp \left(i \tau X_{t}\right)\right)\right|<\infty$, which is necessary for valid Edgeworth expansion establishment. Assumption 2 ensures the integrability of spectral density function and the existence of a positive definite autocovariance function for $\left\{X_{t}\right\}$. By the approximation $\omega^{2 d} \sim$ $|1-\exp (-i \omega)|^{2 d}$ as $\omega \rightarrow 0$, Assumption 2 can be applied to ARFIMA models with spectral density $\frac{\sigma_{\epsilon}^{2}}{2 \pi} \frac{1}{|1-\exp (-i \omega)|^{2 d}} \tilde{f}(\omega)$, for $\tilde{f}(\omega)$ is bounded above and away from zero for all $\omega$.

\subsection{Bartlett correction in Gaussian LMTS}

To study the Bartlett correction of EL, we first establish the stochastic expansion of $l\left(\theta_{0}\right)$. We begin with Lemma 3.1, which evaluates the bias of the estimating equation. For simplicity, define $m_{j}:=m_{j}\left(\theta_{0}\right)$ and for $k=2,3$, 4 , let

$$
\lambda_{k}=\mathrm{E}\left(\frac{1}{n} \sum_{j=1}^{n} m_{j}^{k}\right) \quad \text { and } \quad \Delta_{k}=\frac{1}{n} \sum_{j=1}^{n}\left(m_{j}^{k}-\lambda_{k}\right) .
$$


Lemma 3.1. Under Assumptions 1-2, we have

$$
\mathrm{E}(\bar{m})=O\left(\frac{\log ^{3} n}{n}\right), \quad \text { and } \quad \operatorname{Var}(\bar{m})=O\left(\frac{1}{n}\right) .
$$

Unlike the bias in (2.4) for SMTS of order $n^{-1}$, the larger bias in (3.2) for LMTS results from the dependence of periodogram ordinates at frequencies near origin. However, if the integers $j$ in Fourier frequency $\omega_{j}=2 \pi j / n$ are allowed to increase at a comparable rate with the sample size, i.e., $j / n=j(n) / n \in(0,1]$, then the periodogram ordinates are independently chi-squared distributed (see Lemmas B.2 and B.3). Hence, after some tedious calculations, variance of the estimating function is bounded above by an order $n^{-1}$ quantity. By applying Chebyshev's inequality to (3.2), we have $\bar{m}=O_{p}\left(n^{-1 / 2}\right)$. Together with Lemma B.7 in the Appendix, we establish the stochastic expansion for periodogrambased log-EL ratio,

$$
\begin{aligned}
\frac{1}{n} l\left(\theta_{0}\right)= & \frac{\bar{m}^{2}}{\lambda_{2}}-\frac{\bar{m}^{2} \Delta_{2}}{\lambda_{2}^{2}}+\frac{2}{3} \frac{\lambda_{3} \bar{m}^{3}}{\lambda_{2}^{3}}+\frac{\bar{m}^{2} \Delta_{2}^{2}}{\lambda_{2}^{3}}+\frac{2}{3} \frac{\bar{m}^{3} \Delta_{3}}{\lambda_{2}^{3}}-2 \frac{\lambda_{3} \bar{m}^{3} \Delta_{2}}{\lambda_{2}^{4}} \\
& +\frac{\lambda_{3}^{2} \bar{m}^{4}}{\lambda_{2}^{5}}-\frac{1}{2} \frac{\lambda_{4} \bar{m}^{4}}{\lambda_{2}^{4}}+O_{p}\left(n^{-\frac{5}{2}}\right) .
\end{aligned}
$$

Details to derive this formula are given in the proof of Theorem 3.1. Based on (3.3), the signed root empirical log-likelihood ratio $S R=R_{1}+R_{2}+R_{3}$, where $R_{j}=O_{p}\left(n^{-j / 2}\right)$, can be derived as follows. By collecting the terms of order $O_{p}\left(n^{-1}\right)$ in (3.3) and comparing them to $R_{1}^{2}$, we have

$$
R_{1}=\frac{\bar{m}}{\sqrt{\lambda_{2}}}
$$

By collecting terms of order $O_{p}\left(n^{-3 / 2}\right)$ and comparing them to $2 R_{1} R_{2}$, we have

$$
R_{2}=\frac{1}{3} \frac{\lambda_{3} \bar{m}^{2}}{\lambda_{2}^{5 / 2}}-\frac{1}{2} \frac{\bar{m} \Delta_{2}}{\lambda_{2}^{3 / 2}}
$$

Lastly, by collecting terms of order $O_{p}\left(n^{-2}\right)$ and comparing them to $2 R_{1} R_{3}+R_{2}^{2}$, we have

$$
R_{3}=\frac{3}{8} \frac{\bar{m} \Delta_{2}^{2}}{\lambda_{2}^{5 / 2}}+\frac{1}{3} \frac{\bar{m}^{2} \Delta_{3}}{\lambda_{2}^{5 / 2}}-\frac{5}{6} \frac{\lambda_{3} \bar{m}^{2} \Delta_{2}}{\lambda_{2}^{7 / 2}}+\frac{4}{9} \frac{\lambda_{3}^{2} \bar{m}^{3}}{\lambda_{2}^{9 / 2}}-\frac{1}{4} \frac{\lambda_{4} \bar{m}^{3}}{\lambda_{2}^{7 / 2}} .
$$

Using the above $R_{j}$ forms, Lemma 3.2 gives the asymptotic expansions on the cumulants of $\sqrt{n} S R$.

Lemma 3.2. Let $k_{j}, j=1, \ldots, 4$, be the first four cumulants of $\sqrt{n} S R$. The asymptotic expansion for $k_{j}$ is given by

$$
k_{1}=k_{1,1} \frac{\log ^{3} n}{\sqrt{n}}+k_{1,2} \frac{1}{\sqrt{n}}+k_{1,3} \frac{1}{n}+O\left(\frac{\log ^{6} n}{n^{3 / 2}}\right)
$$




$$
\begin{aligned}
k_{2} & =k_{2,1}+k_{2,2} \frac{\log ^{6} n}{n}+k_{2,3} \frac{\log ^{3} n}{n}+k_{2,4} \frac{1}{n}+O\left(\frac{\log ^{9} n}{n^{2}}\right), \\
k_{3} & =k_{3,1} \frac{1}{\sqrt{n}}+k_{3,2} \frac{1}{n}+O\left(\frac{\log ^{9} n}{n^{3 / 2}}\right), \\
k_{4} & =k_{4,1} \frac{1}{n}+O\left(\frac{\log ^{12} n}{n^{2}}\right),
\end{aligned}
$$

where the coefficients in the asymptotic expansion satisfy

$$
\begin{aligned}
k_{1,1}= & \frac{1}{\sqrt{\lambda_{2}}} \frac{n}{\log ^{3} n} \operatorname{cum}(\bar{m}), \\
k_{1,2}= & \frac{n}{3} \frac{\lambda_{3}}{\lambda_{2}^{5 / 2}} \rho_{11}-\frac{n}{2} \frac{1}{\lambda_{2}^{3 / 2}} \rho_{12}, \\
k_{2,2}= & -\frac{2}{3} \frac{\lambda_{3}}{\lambda_{2}^{3}} \frac{n^{2}}{\log ^{6} n} \operatorname{cum}^{2}(\bar{m}), \\
k_{2,3}= & \frac{n^{2}}{\log ^{3} n}\left(\frac{4}{3} \frac{\lambda_{3}}{\lambda_{2}^{3}} \rho_{11}-\frac{1}{\lambda_{2}^{2}} \rho_{12}\right) \operatorname{cum}(\bar{m}) \\
k_{2,4}= & -\frac{n^{2}}{\lambda_{2}^{2}} \rho_{112}+n^{2} \frac{2}{3} \frac{\lambda_{3}}{\lambda_{2}^{3}} \rho_{111}+n^{2} \frac{7}{4} \frac{1}{\lambda_{2}^{3}} \rho_{12}^{2}-n^{2} \frac{17}{3} \frac{\lambda_{3}}{\lambda_{2}^{4}} \rho_{11} \rho_{12} \\
& +n^{2} \frac{1}{\lambda_{2}^{3}} \rho_{11} \rho_{22}+n^{2} \frac{2}{\lambda_{2}^{3}} \rho_{11} \rho_{13}+n^{2}\left(\frac{26}{9} \frac{\lambda_{3}^{2}}{\lambda_{2}^{5}}-\frac{3}{2} \frac{\lambda_{4}}{\lambda_{2}^{4}}\right) \rho_{11}^{2}, \\
k_{2,1}= & 1, \quad k_{1,3}=k_{3,1}=k_{3,2}=k_{4,1}=0,
\end{aligned}
$$

and $\rho_{u v}$ and $\rho_{u v w}$ are defined as

$$
\begin{aligned}
\rho_{u v} & =\operatorname{cum}\left(\frac{1}{n} \sum_{j=1}^{n} m_{j}^{u}, \frac{1}{n} \sum_{j=1}^{n} m_{j}^{v}\right), \\
\rho_{u v w} & =\operatorname{cum}\left(\frac{1}{n} \sum_{j=1}^{n} m_{j}^{u}, \frac{1}{n} \sum_{j=1}^{n} m_{j}^{v}, \frac{1}{n} \sum_{j=1}^{n} m_{j}^{w}\right) .
\end{aligned}
$$

Note that $k_{u, v} \mathrm{~s}$ are bounded by some constants, for $u, v \in \mathbb{S}_{4}=\{1,2,3,4\}$. Then the cumulants expansions (3.4)-(3.7) lead to the coefficients of polynomials in the Edgeworth expansion for $\sqrt{n} S R$. Due to the larger bias in (3.2), the expansion has an irregular form with a decreasing power series of order $\log ^{3} n / \sqrt{n}$ instead of order $\sqrt{n}$ in weakly dependent case (2.2).

Theorem 3.1. Under Assumptions 1-2, the p.d.f. $\pi(x)$ of $\sqrt{n} S R$ admits a valid Edgeworth expansion

$$
\pi(x)=\phi(x)+\frac{r_{1}(x) \log ^{3} n}{\sqrt{n}} \phi(x)+\frac{r_{2}(x) \log ^{6} n}{n} \phi(x)+O\left(\frac{\log ^{9} n}{n^{3 / 2}}\right),
$$


where

$$
\begin{aligned}
& r_{1}(x)=\frac{\sqrt{n}}{\log ^{3} n}\left\{k_{1} x+\frac{1}{6} k_{3}\left(x^{3}-3 x\right)\right\}, \\
& r_{2}(x)=\frac{n}{\log ^{6} n} \frac{1}{2}\left(k_{2}-1+k_{1}^{2}\right)\left(x^{2}-1\right),
\end{aligned}
$$

and $r_{1}, r_{2}$ are bounded above and below.

With the particular form of Edgeworth expansion in (3.10), calculating the coverage error of EL is equivalent to calculating the integral of the density expansion of signed root decomposition. It is noted that the decreasing series of power $\log ^{3} n / \sqrt{n}$ makes the coverage error larger than conventional one for i.i.d. data.

Theorem 3.2. If Assumptions 1-2 hold, then

$$
P\left(l\left(\theta_{0}\right) \leq c_{\alpha}\right)=1-\alpha+O\left(\frac{\log ^{6} n}{n}\right) .
$$

Theorem 3.2 says that the coverage error of order $\log ^{6} n / n$ is larger for LMTS, which is larger than that of i.i.d. data or short-memory time series. The expectation of the periodogram-based log-EL ratio in this case becomes

$$
\begin{aligned}
\mathrm{E}\left(l\left(\theta_{0}\right)\right)= & \mathrm{E}(\sqrt{n} S R)^{2}+O\left(n^{-3 / 2}\right)=\int_{-\infty}^{\infty} x^{2} \pi(x) d x+O\left(n^{-3 / 2}\right) \\
= & \int_{-\infty}^{\infty}\left\{x^{2} \phi(x)+x^{2} r_{1}(x) \phi(x) \log ^{3} n / \sqrt{n}+x^{2} r_{2}(x) \phi(x) \log ^{6} n / n\right\} d x \\
& +O\left(\log ^{9} n / n^{3 / 2}\right)=1+b \log ^{6} n / n+O\left(\log ^{9} n / n^{3 / 2}\right)
\end{aligned}
$$

where $b=\int_{-\infty}^{\infty} x^{2} r_{2}(x) \phi(x) d x$. The feature that $r_{2}$ has no term of degree 4 or 6 prompts us to scale $l\left(\theta_{0}\right)$ by $1+b \log ^{6} n / n$ for a more accurate approximation. In contrast to short-memory time series, the additional terms $k_{1,1}$ and $k_{1,2}$ in (3.4) in LMTS prevent the coverage error from reducing to $n^{-2}$ via Bartlett correction. However, this scaling adjustment can remove terms involving $k_{2,1}$ and $k_{2,2}$ in (3.5) such that a "slight" Bartlett correction (i.e., from $O\left(\log ^{6} n / n\right)$ to $\left.O\left(\log ^{3} n / n\right)\right)$ can be still achieved.

Theorem 3.3. Define $c_{\alpha}^{*}=\left(1+b \log ^{6} n / n\right) c_{\alpha}$. Under Assumptions $1-2$, it follows that

$$
P\left(l\left(\theta_{0}\right) \leq c_{\alpha}^{*}\right)=1-\alpha+O\left(\frac{\log ^{3} n}{n}\right),
$$

where $b=k_{1,1}^{2}+k_{2,2}$, and $k_{1,1}, k_{2,2}$ are given by (3.8) and (3.9).

In practice, $b$ is unknown, the Bootstrap method in DiCiccio, Hall and Romano [15], Monti [27] and Yau [37] can be used to estimate $b$ from the data. We mention the procedure for the sake of completeness. First, for each series, we calculate the normalized periodogram $\left\{I_{n}\left(\omega_{j}\right) / f\left(\omega_{j}, \theta\right)\right\}$. Since $I_{n}(\pi+\lambda)=$ 
$I_{n}(\pi-\lambda)$, we can restrict our attention to the frequencies $\omega_{j}=2 \pi j / n$ for $j=1,2, \ldots,[(n-1) / 2]$. Define $T=[(n-1) / 2]$, we calculate

$$
y_{j}=\frac{I_{n}\left(\omega_{j}\right)}{f\left(\omega_{j}, \hat{\theta}\right)} / \frac{1}{T} \sum_{l=1}^{T} \frac{I_{n}\left(\omega_{l}\right)}{f\left(\omega_{l}, \hat{\theta}\right)}
$$

where $\hat{\theta}$ is a consistent estimator of $\theta$. Let $F_{T}$ be the empirical distribution function that assigns mass $T^{-1}$ to each $y_{j}$. A bootstrap sample $\left(y_{1}^{a}, y_{2}^{a}, \ldots, y_{T}^{a}\right)$ can be obtained by resampling from $F_{T}$ with replacement. Then, using $I_{n}^{a}\left(\omega_{j}\right)=$ $y_{j}^{a} f\left(\omega_{j}, \hat{\theta}\right)$, we get the resampled periodogram $I_{n}^{a}\left(\omega_{1}\right), I_{n}^{a}\left(\omega_{2}\right), \ldots, I_{n}^{a}\left(\omega_{T}\right)$. Using the resampled periodogram, we compute the periodogram-based log-EL ratio $\left\{\hat{l}\left(\hat{\theta}_{T}\right)\right\}$. The resampling procedure is repeated for $B$ times to obtain a new set $\left\{\hat{l}_{a}\left(\hat{\theta}_{T}\right)\right\}, a=1,2, \ldots, B$. Finally, we estimate the unknown factor $\hat{b}$ by

$$
\frac{1}{B} \sum_{a=1}^{B} \hat{l}_{a}\left(\hat{\theta}_{n}\right)=1+\frac{\hat{b} \log ^{6} n}{n} .
$$

Consequently, the Bartlett corrected confidence interval is given by

$$
\left\{\theta \in \Theta \mid l(\theta) \leq \chi_{1,1-\alpha}^{2}\left(1+\frac{\hat{b} \log ^{6} n}{n}\right)\right\} .
$$

Remark 1. For Gaussian time series, higher order cumulants of the periodogram ordinates can be decomposed into products of the second order cumulants of DFT, where terms with the third or higher order cumulants of DFT can be neglected. However, for non-Gaussian time series, the higher order cumulants of DFT are non-negligible. For example, for two different Fourier frequencies $\omega_{j}$ and $\omega_{k}, j \neq k$ in general,

$$
\begin{aligned}
\operatorname{cum}\left(I_{n}\left(\omega_{j}\right), I_{n}\left(\omega_{k}\right)\right)= & \operatorname{cum}^{2}\left(J_{n}\left(\omega_{j}\right), J_{n}\left(\omega_{k}\right)\right) \operatorname{cum}^{2}\left(J_{n}\left(\omega_{j}\right), J_{n}\left(\omega_{-k}\right)\right) \\
& +\operatorname{cum}\left(J_{n}\left(\omega_{j}\right), J_{n}\left(\omega_{-j}\right), J_{n}\left(\omega_{k}\right), J_{n}\left(\omega_{-k}\right)\right) .
\end{aligned}
$$

For non-Gaussian time series, the fourth order cumulant term in the above equation is non-zero. Therefore, the calculation of higher order cumulants of periodogram ordinates becomes substantially complicated. It is thus much more difficult to establish the valid Edgeworth expansion for the signed root empirical log-likelihood ratio statistic for non-Gaussian processes. In this case, it is unknown whether empirical likelihood is still Bartlett correctable.

\section{Simulation studies}

In this Section, we perform Monte Carlo experiments to demonstrate the Bartlett correction of EL for LMTS models. A simple LMTS model, ARFIMA $(0, d, 0)$, is used. Also, we compare the performance of Whittle likelihood ratio test and Bartlett corrected test, under ARFIMA models. All the simulations in this article are conducted using $\mathrm{R}$ version 2.15.1. 
TABLE 1

Coverage errors of EL and Bartlett corrected EL confidence intervals for ARFIMA $(0, d, 0)$ models, replications $=1,000$

\begin{tabular}{|c|c|c|c|c|c|c|c|c|}
\hline \multicolumn{5}{|c|}{ Two-sided coverage error } & \multicolumn{4}{|c|}{ One-sided coverage error } \\
\hline & $d=0.1$ & $\overline{d e=0.2}$ & $d=0.3$ & $\overline{d e=0.4}$ & $d=0.1$ & $\overline{d d=0.2}$ & 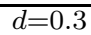 & 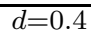 \\
\hline \multicolumn{5}{|c|}{$n=200$} & \multicolumn{4}{|c|}{$n=200$} \\
\hline EL & 0.0469 & 0.0392 & 0.0302 & 0.0393 & 0.0469 & 0.0392 & 0.0302 & 0.0419 \\
\hline Bart. EL & 0.0227 & 0.0169 & 0.0067 & 0.0527 & 0.0227 & 0.0169 & 0.0112 & 0.0717 \\
\hline \multicolumn{4}{|c|}{$n=1,000$} & & \multicolumn{4}{|c|}{$n=1,000$} \\
\hline EL & 0.036 & 0.01 & 0.008 & 0.02 & 0.036 & 0.028 & 0.03 & 0.02 \\
\hline Bart. EL & 0.0223 & 0.008 & 0.003 & 0.023 & 0.0223 & 0.024 & 0.027 & 0.023 \\
\hline \multicolumn{4}{|c|}{$n=1,500$} & & \multicolumn{4}{|c|}{$n=1,500$} \\
\hline EL & 0.028 & 0.003 & 0.006 & 0.025 & 0.028 & 0.007 & 0.008 & 0.025 \\
\hline Bart. EL & 0.021 & 0.001 & 0.002 & 0.022 & 0.021 & 0.007 & 0.004 & 0.022 \\
\hline
\end{tabular}

The ARFIMA $(p, d, q)$ process $X_{t}$ with memory parameter $d$ is given by

$$
\phi(B) X_{t}=(1-B)^{-d} \psi(B) \epsilon_{t}, \quad \epsilon_{t} \stackrel{i . i . d .}{\sim} N(0,1),
$$

where $N(0,1)$ denotes the standard normal distribution with mean 0 and variance $1, \psi(B)=\left(1-\psi_{1} B-\cdots-\psi_{q} B^{q}\right)$ and $\phi(B)=\left(1-\phi_{1} B-\cdots-\phi_{p} B^{p}\right)$.

Recall that the asymptotic $1-\alpha$ confidence interval for $d=\theta / 2$ is given by $\mathbf{I}_{\alpha}(d)=\left\{d \mid l(d) \leq c_{\alpha}\right\}$ and the Bartlett corrected confidence interval is $\mathbf{I}_{\alpha}^{\prime}(d)=\left\{d \mid l(d) \leq c_{\alpha}^{*}\right\}$. To construct $\mathbf{I}_{\alpha}(d)$ and $\mathbf{I}_{\alpha}^{\prime}(d)$, we substitute various values of $d$ into the $\log$-EL ratio and compare them with the critical value $c_{\alpha}$. The simulations are conducted for $d_{0}=0.1,0.2,0.3,0.4$. In Table 1 , we compare $E_{\alpha}$ and $E_{\alpha}^{\prime}$ for $\alpha=0.05$ and sample sizes $n=200,1,000$ and 1,500. In each case, 1,000 replications are drawn. In the procedure of Bootstrap sampling, we adopt the Whittle maximum likelihood estimator as the consistent estimator and set the resampling replications $B$ to be 500 .

We use the coverage error to evaluate the performance of the asymptotic distribution confidence intervals. Let $d_{0}, d_{[\alpha / 2]}$ and $d_{[1-\alpha / 2]}$ be the true value of the parameter, the lower and the upper endpoints of the confidence interval, respectively. The one-sided and two-sided coverage error are defined by

$$
\left|P\left\{d_{0}<d_{[\alpha / 2]}\right\}-\alpha / 2\right|+\left|P\left\{d_{0}>d_{[1-\alpha / 2]}\right\}-\alpha / 2\right|,
$$

and

$$
\left|P\left\{\left(d_{0}<d_{[\alpha / 2]}\right) \cup\left(d_{0}>d_{[1-\alpha / 2]}\right)\right\}-\alpha\right| .
$$

The simulations show that the coverage accuracy of both confidence intervals is higher for larger $n$, which supports that both of the two statistics tend to a $\chi_{1}^{2}$ variate. Also, except for $d=0.4$ and $n \leq 1,000$, Bartlett corrected intervals have smaller coverage error than the non-Bartlett corrected counterparts. Figures 1a1c present the QQ plot between the log-EL ratio and Bartlett corrected log-EL ratio and the $\chi_{1}^{2}$ random variable. The closer the lines to the $45^{\circ}$ straight line, the more accurate the corresponding asymptotic distribution. As it is found that for large $n$, the asymptotic accuracy of both the EL and the Bartlett corrected 


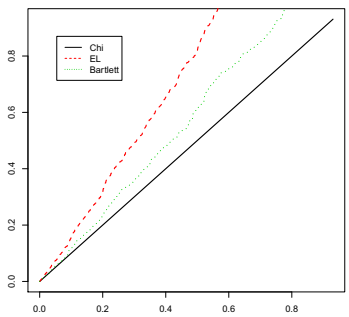

(a) $n=50$ and $H_{0}: d=0.1$.

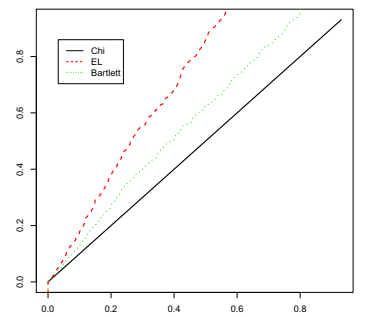

(b) $n=50$ and $H_{0}: d=0.2$.

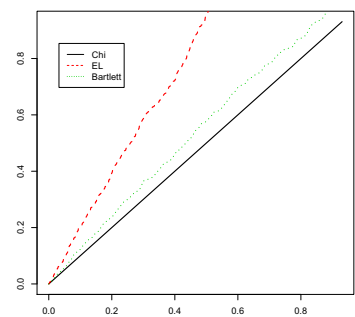

(c) $n=25$ and $H_{0}: d=0.1$.

FIG 1. QQ plot for Bartlett correction in the ARFIMA $(0, d, 0)$ models.

TABLE 2

Coverage errors of Whittle and Bartlett corrected confidence intervals for ARFIMA $(0, d, 0)$ models, replications $=1,000$

\begin{tabular}{|c|c|c|c|c|c|c|c|c|}
\hline \multicolumn{5}{|c|}{ Two-sided coverage error } & \multicolumn{4}{|c|}{ One-sided coverage error } \\
\hline & $d=0.1$ & $\overline{d=0.2}$ & $d=0.3$ & $d=0.4$ & $d=0.1$ & $d=0.2$ & $\overline{d=0.3}$ & $d=0.4$ \\
\hline \multicolumn{5}{|c|}{$n=200$} & \multicolumn{4}{|c|}{$n=200$} \\
\hline EL & 0.011 & 0.006 & 0.007 & 0.019 & 0.011 & 0.006 & 0.007 & 0.019 \\
\hline Bart. EL & 0.034 & 0.051 & 0.053 & 0.057 & 0.041 & 0.051 & 0.053 & 0.057 \\
\hline \multicolumn{5}{|c|}{$n=1,000$} & \multicolumn{4}{|c|}{$n=1,000$} \\
\hline EL & 0.003 & 0.002 & 0.001 & 0.012 & 0.03 & 0.002 & 0.003 & 0.012 \\
\hline Bart. EL & 0.007 & 0.01 & 0.006 & 0.015 & 0.03 & 0.04 & 0.044 & 0.033 \\
\hline
\end{tabular}

EL are very similar, we only show the cases with small sample sizes. It can be seen that the Bartlett correction does improve the approximation.

Alternatively, we may use Whittle likelihood ratio test to construct the confidence interval, because Hosoya [21] proved for LMTS that

$$
-2\left\{W L\left(d_{0}\right)-W L(\hat{d})\right\} \stackrel{d}{\rightarrow} \chi_{1}^{2},
$$

where $\hat{d}$ is a consistent estimator of $d$. However, Bartlett correction does not work for Whittle likelihood ratio statistic in finite sample performance. To show this, we further compare coverage error of Whittle likelihood ratio test and Bartlett corrected test with $\alpha=0.05$ and sample sizes $n=50,200$ and 500. In calculating the Bartlett correction factor step, we also adopt the Whittle estimator as the consistent estimator, and the Bootstrap iteration is set to 500 times.

The simulation results in Table 2 show that the Whittle likelihood ratio converges to chi-squared random variable as sample size increases. However, the Bartlett corrected coverage error does not converge to zero in general. But this technique fails to improve the conventional Whittle likelihood ratio test in almost all cases. This feature provides our periodogram-based EL superior in real applications.

To explore the applicability of Bartlett correction for non-Gaussian LMTS, we conducted a small scale simulation studies on the coverage accuracies of Bartlett corrected EL confidence intervals for $\operatorname{ARFIMA}(0, d, 0)$ models with $t_{5}$ and $\operatorname{Exp}(1)$ white noise process. We considered nominal coverage error of 
TABLE 3

Two-sided coverage errors of Bartlett corrected EL confidence intervals for ARFIMA $(0, d, 0)$ models with non-Gaussian noises, replications $=1,000$

\begin{tabular}{|c|c|c|c|c|c|c|c|c|}
\hline \multicolumn{5}{|c|}{$t_{5}$} & \multicolumn{4}{|c|}{$\operatorname{Exp}(1)$} \\
\hline & $\overline{d e=0.1}$ & $\overline{d=0.2}$ & $\overline{d e=0.3}$ & $\overline{d e=0.4}$ & $\overline{d e=0.1}$ & $\overline{d e=0.2}$ & $\overline{d e=0.3}$ & $\overline{d \bar{d}=0.4}$ \\
\hline \multicolumn{5}{|c|}{$n=200$} & \multicolumn{4}{|c|}{$n=200$} \\
\hline EL & 0.047 & 0.046 & 0.038 & 0.062 & 0.037 & 0.042 & 0.045 & 0.04 \\
\hline Bart. EL & 0.035 & 0.039 & 0.036 & 0.06 & 0.032 & 0.029 & 0.027 & 0.045 \\
\hline \multicolumn{5}{|c|}{$n=1,000$} & \multicolumn{4}{|c|}{$n=1,000$} \\
\hline EL & 0.027 & 0.033 & 0.02 & 0.028 & 0.03 & 0.017 & 0.012 & 0.02 \\
\hline Bart. EL & 0.011 & 0.025 & 0.012 & 0.021 & 0.028 & 0.011 & 0.003 & 0.006 \\
\hline
\end{tabular}

$\alpha=0.05$ and sample sizes of $n=200$ and 1,000. The results are summarized in Table 3. It can be seen that the coverage errors of EL confidence intervals can be reduced by Bartlett correction even for non-Gaussian noises.

\section{Acknowledgements}

We would like to thank the Editor and the anonymous referee for helpful comments, which led to an improved version of this paper. This research was supported in part by grants from the General Research Fund of HKSAR-RGCGRF Nos 400313, 400410 and HKSAR-RGC-CRF: CityU8/CRG/12G (Chan), HKSAR-RGC-ECS 405012, HKSAR-RGC-GRF 405113 (Yau). Research support from the Statistics Department of CUHK is gratefully acknowledged.

\section{Appendix A: Proofs of theorems}

Proof of Theorem 3.1. In order to study Edgeworth expansion of density for signed root empirical log-likelihood ratio, we need to develop stochastic expansion for $l\left(\theta_{0}\right)$. First, we establish that $t$ is small of order $O_{p}\left(n^{-1 / 2}\right)$. For the score function $m_{j}=m\left(X_{j}, \theta_{0}\right)$ evaluated at the true value $\theta_{0}, t$ is the solution to the equation

$$
\frac{1}{n} \sum_{j=1}^{n} \frac{m_{j}}{1+t m_{j}}=0
$$

Substituting $1 /\left(1+t m_{j}\right)=1-t m_{j} /\left(1+t m_{j}\right)$ into (A.1) and simplifying, we have

$$
\frac{1}{n} \sum_{j=1}^{n} m_{j}=\frac{1}{n} \sum_{j=1}^{n} \frac{t m_{j}^{2}}{1+t m_{j}} .
$$

Since $p_{j}>0$ and $p_{j}=\frac{1}{n} \frac{1}{1+t m_{j}}$, we have $1+t m_{j}>0$, and

$$
t \frac{1}{n} \sum_{j=1}^{n} m_{j}^{2} \leq t \frac{1}{n} \sum_{j=1}^{n} \frac{m_{j}^{2}}{1+t m_{j}}\left(1+\max _{1 \leq j \leq n} t m_{j}\right)
$$




$$
=\frac{1}{n} \sum_{j=1}^{n} m_{j}\left(1+t \max _{1 \leq j \leq n} m_{j}\right)
$$

where the equality in (A.3) follows from (A.2). By Lemma 3.1, we have

$$
\frac{1}{n} \sum_{j=1}^{n} m_{j}=O_{p}\left(n^{-1 / 2}\right)
$$

By Lemma B.7 in Appendix B, we have

$$
\frac{1}{n} \sum_{j=1}^{n} m_{j}^{2}=\lambda_{2}+O_{p}\left(n^{-1 / 2}\right)=O_{p}(1)
$$

Also, from Hong-Zhi An et al. [2], we obtain

$$
\begin{aligned}
\max _{1 \leq j \leq n} m_{j} & \leq \max _{1 \leq j \leq n}\left(\frac{\partial}{\partial \theta} \log f\left(\omega_{j}, \theta\right)\right) \max _{1 \leq j \leq n}\left(\frac{I_{n}\left(\omega_{j}\right)}{f\left(\omega_{j}, \theta\right)}\right) \\
& =O(\log n) O_{p}(\log n)=O_{p}\left(\log ^{2} n\right) .
\end{aligned}
$$

In conclusion, combining (A.3), (A.4), (A.5) and (A.6), it can be seen that

$$
t=O_{p}\left(n^{-1 / 2}\right)
$$

Applying Taylor expansion to (A.1), we get

$$
\frac{1}{n} \sum_{j=1}^{n} m_{j}\left(1-t m_{j}+\left(t m_{j}\right)^{2}+\cdots\right)=0
$$

Solving for $t$, it follows that

$t=\frac{\bar{m}}{\lambda_{2}}-\frac{\bar{m} \Delta_{2}}{\lambda_{2}^{2}}+\frac{\lambda_{3} \bar{m}^{2}}{\lambda_{2}^{3}}+\frac{\bar{m} \Delta_{2}^{2}}{\lambda_{2}^{3}}-3 \frac{\lambda_{3} \bar{m}^{2} \Delta_{2}}{\lambda_{2}^{4}}+2 \frac{\lambda_{3}^{2} \bar{m}^{3}}{\lambda_{2}^{5}}+\frac{\bar{m}^{2} \Delta_{3}}{\lambda_{2}^{3}}-\frac{\lambda_{4} \bar{m}^{3}}{\lambda_{2}^{4}}+O_{p}\left(n^{-2}\right)$.

Note that $t=O_{p}\left(n^{-1 / 2}\right)$. Substituting $t$ into (1.2), we have the stochastic expansion (3.3). Then $R_{j}$ s and asymptotic expansions of $k_{j}$ s are obtained as discussed in Section 3. The characteristic function of $\sqrt{n} S R$ is thus given by

$$
\begin{aligned}
\psi_{n}(x) & =\exp \left\{k_{1}(i x)+\frac{1}{2} k_{2}(i x)^{2}+O\left(\frac{\log ^{9} n}{n^{3 / 2}}\right)\right\} \\
& =e^{-\frac{x^{2}}{2}}\left\{1+k_{1}(i x)+\frac{1}{2}\left(k_{2}-1+k_{1}^{2}\right)(i x)^{2}\right\}+O\left(\frac{\log ^{9} n}{n^{3 / 2}}\right) .
\end{aligned}
$$

Applying Fourier inversion formula to $\psi_{n}(x)=\int_{-\infty}^{\infty} e^{i \tau x} \pi(x) d \tau, \pi(x)$ admits the Edgeworth expansion (3.10). This completes the proof of Theorem 3.1. 
Proof of Theorem 3.2. From the Edgeworth expansion (3.10) of $\pi(x)$, we have

$$
\begin{aligned}
& P\left(l\left(\theta_{0}\right) \leq c_{\alpha}\right) \\
= & \left.P\left(n S R^{2}+R_{n}\right) \leq c_{\alpha}\right)=\int_{-\sqrt{c_{\alpha}}}^{\sqrt{c_{\alpha}}} \phi(x) d x+\int_{-\sqrt{c_{\alpha}}}^{\sqrt{c_{\alpha}}} k_{1} H_{1}(x) \phi(x) d x \\
& +\int_{-\sqrt{c_{\alpha}}}^{\sqrt{c_{\alpha}}}\left(\frac{1}{2}\left(k_{2}-1+k_{1}^{2}\right) H_{2}(x)\right) \phi(x) d x+O\left(\frac{\log ^{9} n}{n^{3 / 2}}\right) \\
= & 1-\alpha+O\left(\frac{\log ^{6} n}{n}\right),
\end{aligned}
$$

where $H_{j}(x), j=1, \ldots, 6$ are Hermite polynomials [18]. The order $O\left(\log ^{6} n / n\right)$ of error term comes from $k_{2}-1+k_{1}^{2}$. This completes the proof of Theorem 3.2.

Proof of Theorem 3.3. The proof relies on the Edgeworth expansion of density for the corrected signed root empirical log-likelihood ratio, i.e., $S R^{*}=S R(1-$ $\left.\frac{b \log ^{6} n}{2 n}\right)$, which requires the asymptotic expansion of the cumulants of $\sqrt{n} S R^{*}$. Scaling the log-EL ratio by its mean, we have

$$
\begin{aligned}
& l\left(\theta_{0}\right) /\left(1+b \log ^{6} n / n\right)=\left\{\sqrt{n} S R\left(1-\frac{b \log ^{6} n}{2 n}\right)\right\}^{2}+O_{p}\left(n^{-3 / 2}\right) \\
= & \left(\sqrt{n} S R^{*}\right)^{2}+O_{p}\left(n^{-3 / 2}\right) .
\end{aligned}
$$

Using this equation, we can deduce the asymptotic expansion of $k_{j}^{*}, j=1, \ldots, 4$, which are the first four cumulants of $\sqrt{n} S R^{*}$,

$$
\begin{gathered}
k_{1}^{*}=\frac{k_{1,1} \log ^{3} n}{\sqrt{n}}+\frac{k_{1,2}}{n^{1 / 2}}+O\left(\frac{\log ^{9} n}{n^{3 / 2}}\right), \quad k_{2}^{*}=1-\frac{k_{1,1}^{2} \log ^{6} n}{n}+\frac{k_{2,3} \log ^{3} n}{n}+O\left(\frac{1}{n}\right), \\
k_{3}^{*}=\frac{k_{3,1}}{\sqrt{n}}+O\left(\frac{\log ^{6} n}{n^{3 / 2}}\right), \quad k_{4}^{*}=\frac{k_{4,1}}{n}+O\left(\frac{\log ^{12} n}{n^{2}}\right) .
\end{gathered}
$$

Thus, the p.d.f. $\pi^{*}(x)$ of $\sqrt{n} S R^{*}$ admits an Edgeworth expansion

$$
\pi^{*}(x)=\phi(x)+\frac{r_{1}^{*}(x) \log ^{3} n}{\sqrt{n}} \phi(x)+\frac{r_{2}^{*}(x) \log ^{6} n}{n} \phi(x)+O\left(\frac{\log ^{9} n}{n^{3 / 2}}\right),
$$

where

$$
\begin{aligned}
& r_{1}^{*}(x)=\frac{\sqrt{n}}{\log ^{3} n}\left[k_{1}^{*} H_{1}(x)+\frac{1}{6} k_{3}^{*} H_{3}(x)\right], \\
& r_{2}^{*}(x)=\frac{n}{\log ^{6} n}\left[\frac{1}{2}\left(k_{2}^{*}-1+\left(k_{1}^{*}\right)^{2}\right) H_{2}(x)+\left(\frac{k_{4}^{*}}{24}+\frac{k_{1}^{*} k_{3}^{*}}{6}\right) H_{4}(x)+\frac{\left(k_{3}^{*}\right)^{2}}{72} H_{6}(x)\right] .
\end{aligned}
$$


The coefficients of Hermite polynomials in $r_{2}^{*}(x)$ satisfy

$$
\begin{aligned}
k_{2}^{*}-1+\left(k_{1}^{*}\right)^{2} & =\left(2 k_{1,1} k_{1,2}+k_{2,3}\right) \frac{\log ^{3} n}{n}+O\left(\frac{1}{n}\right), \\
\frac{1}{6} k_{1}^{*} k_{3}^{*}+\frac{1}{24} k_{4}^{*} & =\frac{k_{1,1} k_{3,1}}{6} \frac{\log ^{3} n}{n}+\frac{k_{4,1}}{24} \frac{1}{n}+O\left(\frac{\log ^{9} n}{n^{3 / 2}}\right), \\
\frac{1}{72}\left(k_{3}^{*}\right)^{2} & =\frac{k_{3,1}^{2}}{72} \frac{1}{n}+O\left(\frac{\log ^{6} n}{n^{2}}\right) .
\end{aligned}
$$

In the Appendix $\mathrm{C}$, we show that $k_{3,1}=k_{4,1}=0$. Hence, $\frac{1}{6} k_{1}^{*} k_{3}^{*}+\frac{1}{24} k_{4}^{*}$ and $\frac{1}{72}\left(k_{3}^{*}\right)^{2}$ are bounded by $O\left(\log ^{3} n / n\right)$. Consequently, after a standard argument, the coverage probability follows

$$
P\left(l\left(\theta_{0}\right) \leq c_{\alpha}^{*}\right)=1-\alpha+O\left(\frac{\log ^{3} n}{n}\right),
$$

completing the proof of Theorem 3.3.

\section{Appendix B: Technical lemmas for cumulant calculations}

Let $\delta \in(0,1)$ and $\epsilon>0$ be generic constants. Define the discrete Fourier transform (DFT) $J_{n}\left(\omega_{j}\right)$ as

$$
J_{n}\left(\omega_{j}\right)=\frac{1}{\sqrt{2 \pi n}} \sum_{t=1}^{n} X_{t} e^{-i t \omega_{j}} .
$$

Note that $I_{n}\left(\omega_{j}\right)=J_{n}\left(\omega_{j}\right) J_{n}\left(\omega_{-j}\right)$ and $J_{n}\left(\omega_{j}\right) / \sqrt{f\left(\omega_{j}\right)}$ is referred as the normalized DFT. To calculate $\sum_{j=1}^{n} R\left(I_{n}\left(\omega_{j}\right) / f\left(\omega_{j}\right)\right)$ for some functions $R$, we need to study the limiting covariance of the normalized DFT for the whole frequency domain. Define $\Lambda=\left\{j \in \mathbb{S}_{n}: \delta n \leq j \leq n\right\}$, then $\omega_{j}$ in this region are bounded away from zero. The whole frequency region on the plane $(0,2 \pi) \times(0,2 \pi)$ can be partitioned into four complementary regions

$$
\begin{aligned}
& \Lambda_{1}=\left\{(j, k): j, k \in \Lambda ; j \neq k ;\left|\omega_{j}-\omega_{k}\right| \leq \epsilon\right\}, \\
& \Lambda_{2}=\left\{(j, k): j, k \in \Lambda ; \epsilon<\left|\omega_{j}-\omega_{k}\right|<2 \pi\right\}, \\
& \Lambda_{3}=\left\{(j, k): j \in \mathbb{S}_{n}, k \in \Lambda ;\left|\omega_{j}\right| \leq \epsilon\right\} \cup\left\{(j, k): k \in \mathbb{S}_{n}, j \in \Lambda ;\left|\omega_{k}\right| \leq \epsilon\right\}, \\
& \Lambda_{4}=\left\{(j, k): j, k \in \mathbb{S}_{n} ;\left|\omega_{j}\right| \leq \epsilon ;\left|\omega_{k}\right| \leq \epsilon ; j \neq k\right\} .
\end{aligned}
$$

Proof of Lemma 3.1. For any constants $K$ and any small $\delta>0$, the expectation of $\bar{m}$ are divided into three regions as

$$
\begin{aligned}
\mathrm{E}(\bar{m}) & =\frac{1}{n} E\left(\sum_{j=1}^{[\log \log n]} m_{j}+\sum_{j=[\log \log n]+1}^{[\delta n]} m_{j}+\sum_{j=[\delta n]+1}^{n} m_{j}\right) \\
& \leq K \frac{\log ^{1+\delta} n}{n}+\frac{K}{n} \sum_{j=[\log \log n]+1}^{[\delta n]} \frac{\log j}{j} \log \left(\frac{j}{n}\right)+\frac{K}{n} \sum_{j=[\delta n]+1}^{n} \frac{\log n}{n},
\end{aligned}
$$


where $[x]$ is the largest integer that is less than or equals to $x$. For the first term of (B.1), $m_{j}=O(\log n)$ since $\frac{\partial}{\partial \theta} \log f\left(\omega_{j}\right) \sim \log n$, and $I_{n}\left(\omega_{j}\right) / f\left(\omega_{j}\right)-1=O(1)$ by Lemma B.4. By the formula

$$
\sum_{j=1}^{n} \frac{\log j}{j}=\frac{1}{2} \log ^{2} n+O\left(\frac{\log ^{2} n}{n}\right)
$$

and Lemma B.5, the second term in (B.1) is of order $O\left(\log ^{3} n / n\right)$. Additionally, using Lemma B.2, the last term is of order $O(\log n / n)$. Summing these parts, $\mathrm{E}(\bar{m})=O\left(\log ^{3} n / n\right)$. For the variance of $\bar{m}$, note that

$$
\operatorname{Var}(\bar{m})=P_{1}+P_{2},
$$

where

$$
P_{1}=\frac{1}{n^{2}} \sum_{j=1}^{n} \operatorname{Var}\left(m_{j}\right), \quad P_{2}=\frac{1}{n^{2}} \sum_{j \neq k} \operatorname{Cov}\left(m_{j}, m_{k}\right) .
$$

Then by Lemmas B.2 and B.3,

$$
\begin{aligned}
& P_{1} \\
= & \frac{1}{n^{2}} \sum_{j=1}^{n}\left(\frac{\partial}{\partial \theta} \log f\left(\omega_{j}\right)\right)^{2}\left[\mathrm{E}^{2}\left(\frac{J_{n}\left(\omega_{j}\right) J_{n}\left(\omega_{-j}\right)}{\sqrt{f\left(\omega_{j}\right) f\left(\omega_{-j}\right)}}\right)+\mathrm{E}\left(\frac{J_{n}^{2}\left(\omega_{j}\right)}{f\left(\omega_{j}\right)}\right) \mathrm{E}\left(\frac{J_{n}^{2}\left(\omega_{-j}\right)}{f\left(\omega_{-j}\right)}\right)\right] \\
\leq & \frac{K}{n^{2}}\left[\sum_{j=1}^{[\log \log n]} \log ^{2} n+\sum_{j=[\log \log n]+1}^{[\delta n]}(\log j-\log n)^{2}\left(1+\frac{\log j}{j}\right)^{2}\right. \\
& \left.+\sum_{j=[\delta n]+1}^{n}\left(1+O\left(\frac{\log n}{n}\right)\right)^{2}\right]=O\left(n^{-1}\right),
\end{aligned}
$$

and

$$
\begin{aligned}
P_{2}= & \frac{1}{n^{2}} \sum_{j \neq k}\left(\frac{\partial}{\partial \theta} \log f\left(\omega_{j}\right)\right)\left(\frac{\partial}{\partial \theta} \log f\left(\omega_{k}\right)\right) \\
& \times\left[\mathrm{E}^{2}\left(\frac{J_{n}\left(\omega_{j}\right) J_{n}\left(\omega_{-k}\right)}{\sqrt{f\left(\omega_{j}\right) f\left(\omega_{-k}\right)}}\right)+\mathrm{E}^{2}\left(\frac{J_{n}\left(\omega_{j}\right) J_{n}\left(\omega_{k}\right)}{\sqrt{f\left(\omega_{j}\right) f\left(\omega_{k}\right)}}\right)\right] \\
= & \frac{1}{n^{2}}\left[\sum_{\left|\omega_{j}-\omega_{k}\right| \leq \epsilon}+\sum_{\left|\omega_{j}-\omega_{k}\right| \geq \epsilon}\right] .
\end{aligned}
$$

Considering the two parts separately, it follows that

$$
\frac{1}{n^{2}} \sum_{\left|\omega_{j}-\omega_{k}\right| \leq \epsilon}=\frac{1}{n^{2}}\left\{\sum_{j, k \in \Lambda_{4}}+\sum_{j, k \in \Lambda_{1}}\right\}
$$




$$
\begin{aligned}
\leq & \frac{K}{n^{2}}\left\{\sum_{1 \leq k \leq[\log \log n] \leq j \leq \delta n} \log n(\log j-\log n)\left(\frac{\log j}{k}\right)^{2}\right. \\
& \left.+\sum_{j=[\log \log n]+1} \sum_{k=[\log \log n]}^{j} \log ^{2} n\left(\frac{\log j}{k}\right)^{2}+(1-\delta)^{2} n^{2}\left(\frac{\log ^{2} n}{n^{2}}\right)\right\} \\
= & o\left(n^{-1}\right),
\end{aligned}
$$

and

$$
\begin{aligned}
\frac{1}{n^{2}} \sum_{\left|\omega_{j}-\omega_{k}\right| \geq \epsilon}= & \frac{1}{n^{2}}\left\{\sum_{j, k \in \Lambda_{2}}+\sum_{j, k \in \Lambda_{3}}\right\} \\
\leq & \frac{K}{n^{2}}\left\{\sum_{j=1}^{[\delta n]}(\log j-\log n)^{2}(1-\delta) n\left(\frac{\log ^{2} n}{n^{2}}\right)\right. \\
& \left.+\sum_{k=1}^{[\delta n]}(\log k-\log n)^{2}(1-\delta) n\left(\frac{\log ^{2} n}{n^{2}}\right)+(1-\delta)^{2} n^{2}\left(\frac{\log ^{2} n}{n^{2}}\right)\right\} \\
& =o\left(n^{-1}\right) .
\end{aligned}
$$

Thus, $P_{2}=o\left(n^{-1}\right)$ and $\operatorname{Var}(\bar{m})=P_{1}+P_{2}=O\left(n^{-1}\right)$.

Next we state some technical Lemmas for the cumulant expansions. The proofs can be found in the Appendix C.

\section{Lemma B.1.}

$$
\frac{1}{n} \int_{-\pi}^{\pi}\left|\frac{\sin (n \mu / 2)}{\sin (\mu / 2)}\right| d \mu \sim \frac{1}{\pi} \frac{\log n}{n} \quad \text { as } n \rightarrow \infty .
$$

Lemma B.2. For any sequences of integers $j=j(n)$ with $j \in \Lambda$, we have

$$
\mathrm{E}\left(\frac{J_{n}\left(\omega_{j}\right) J_{n}\left(\omega_{-j}\right)}{\sqrt{f\left(\omega_{j}\right) f\left(\omega_{-j}\right)}}\right)=1+O\left(\frac{\log n}{n}\right), \quad \text { and } \quad \mathrm{E}\left(\frac{J_{n}^{2}\left(\omega_{j}\right)}{f\left(\omega_{j}\right)}\right)=O\left(\frac{\log n}{n}\right) .
$$

Lemma B.3. For any two sequences of integers $j=j(n)$ and $k=k(n)$ such that $\{j, k\} \in \Lambda_{1} \cup \Lambda_{2} \cup \Lambda_{3}$, we have

$\mathrm{E}\left(\frac{J_{n}\left(\omega_{j}\right) J_{n}\left(\omega_{-k}\right)}{\sqrt{f\left(\omega_{j}\right) f\left(\omega_{-k}\right)}}\right)=O\left(\frac{\log n}{n}\right), \quad$ and $\quad \mathrm{E}\left(\frac{J_{n}\left(\omega_{j}\right) J_{n}\left(\omega_{k}\right)}{\sqrt{f\left(\omega_{j}\right) f\left(\omega_{k}\right)}}\right)=O\left(\frac{\log n}{n}\right)$.

Lemma B.4 and B.5 describe the different behaviors of the expectation of a product of DFTs under Fourier frequencies $\omega_{j}$ with fixed $j$ and slowly increasing $j$, respectively. 
Lemma B.4 (P.M. Robinson [35]). For $0<|d|<\frac{1}{2}$ and any fixed integers $j \neq k, b_{d}=2 \Gamma(1-2 d) \sin \pi d$, we have

$\lim _{n \rightarrow \infty} \mathrm{E}\left(\frac{I_{n}\left(\omega_{j}\right)}{f\left(\omega_{j}\right)}\right)=\frac{b_{d}|j|^{2 d}}{(2 \pi)^{1-2 d}}\left\{4 \int_{0}^{1} u^{2 d-1}(u-1) \sin ^{2}(\pi j u) d u+\frac{1}{d(2 d+1)}\right\}$,

and

$$
\lim _{n \rightarrow \infty} \mathrm{E}\left(\frac{J_{n}\left(\omega_{j}\right) J_{n}\left(\omega_{k}\right)}{\sqrt{f\left(\omega_{j}\right) f\left(\omega_{k}\right)}}\right)=P_{d}(j, k),
$$

where

$$
P_{d}(j, k)=\frac{-2 b_{d}|j k|^{d}}{(2 \pi)^{1-2 d}(j+k)} \int_{0}^{1} u^{2 d-1}\{\sin (2 \pi j u)+\sin (2 \pi k u)\} d u .
$$

In particular, if the white noise process is Gaussian and $j \pm k \neq 0$, then

$$
\begin{gathered}
\lim _{n \rightarrow \infty} \mathrm{E}\left(\frac{I_{n}\left(\omega_{j}\right)}{f\left(\omega_{j}\right)}\right)=2 P_{d}^{2}(j, j), \\
\lim _{n \rightarrow \infty} \operatorname{Cov}\left(\frac{I_{n}\left(\omega_{j}\right)}{f\left(\omega_{j}\right)}, \frac{I_{n}\left(\omega_{k}\right)}{f\left(\omega_{k}\right)}\right)=P_{d}^{2}(j, k)+P_{d}^{2}(j,-k) .
\end{gathered}
$$

Lemma B.5 (P.M. Robinson [35]). Under Assumptions 1-2, for sequences of positive integers $j, k$ that satisfy $K \log \log n<k<j<\delta n$, we have

$$
\begin{aligned}
& E\left(\frac{J_{n}\left(\omega_{j}\right) J_{n}\left(\omega_{-j}\right)}{\sqrt{f\left(\omega_{j}\right) f\left(\omega_{-j}\right)}}\right)=1+O\left(\frac{\log j}{j}\right), \\
& E\left(\frac{J_{n}\left(\omega_{j}\right) J_{n}\left(\omega_{j}\right)}{\sqrt{f\left(\omega_{j}\right) f\left(\omega_{j}\right)}}\right)=O\left(\frac{\log j}{j}\right), \\
& E\left(\frac{J_{n}\left(\omega_{j}\right) J_{n}\left(\omega_{k}\right)}{\sqrt{f\left(\omega_{j}\right) f\left(\omega_{k}\right)}}\right)=O\left(\frac{\log j}{k}\right), \\
& E\left(\frac{J_{n}\left(\omega_{j}\right) J_{n}\left(\omega_{-k}\right)}{\sqrt{f\left(\omega_{j}\right) f\left(\omega_{-k}\right)}}\right)=O\left(\frac{\log j}{k}\right) .
\end{aligned}
$$

The following Lemma gives the lower bound for the covariance of DFT at conjugate frequency in the first case of Lemma B.5.

Lemma B.6. Under Assumptions 1-2, for sequences of positive integers $j$, satisfying $0<K \log \log n<j<\delta n$, we have

$$
\mathrm{E}\left(\frac{J_{n}\left(\omega_{j}\right) J_{n}\left(\omega_{-j}\right)}{\sqrt{f\left(\omega_{j}\right) f\left(\omega_{-j}\right)}}-1\right) \geq K \frac{1}{j} .
$$

Remark. The side lobes of the Fejér kernel in the range $[2 \pi / n, \infty)$ makes it difficult to evaluate the exact magnitude of the integral in $\left[2 \pi / n, \omega_{j}\right]$ when $\omega_{j}$ is not a fixed constant. Using the properties of Dirichlet kernel, Robinson [35] only derives an upper bound for the bias $\mathrm{E}\left(I_{n}\left(\omega_{j}\right) / f\left(\omega_{j}\right)\right)-1$, and the order 
$O\left(\log ^{3} n / n\right)$ in (3.2) are actually an upper bound of the $\mathrm{E}(\bar{m})$. Using order $j^{-1}$ in (B.3), we can find the lower bound $O\left(\log ^{2} n / n\right)$ for $\mathrm{E}(\bar{m})$. It follows that lower bound of the coverage error is reduced from order $O_{p}\left(\log ^{4} n / n\right)$ to order $O_{p}\left(\log ^{2} n / n\right)$ after the Bartlett Correction. This argument justifies the slight Bartlett correction in improving the coverage accuracy even for LMTS.

The following Lemma provides the key order magnitude to derive the asymptotic expansion of $t$ in Lemma 3.1 and $l\left(\theta_{0}\right)$ in (3.3).

Lemma B.7. Under Assumptions 1-2, we have

$$
\lambda_{k}=O(1), \quad \Delta_{k}=O_{p}\left(\frac{1}{\sqrt{n}}\right), \quad \text { for } \quad k=2,3,4,
$$

where $\lambda_{k}$ and $\Delta_{k}$ are defined in (3.1).

Proof of Lemma B.7. Under the Gaussian assumption, the cumulants of normalized DFT with order 3 or higher vanish, so it suffices to consider the products of second order cumulants. Together with the cumulant expansion formula in Brillinger [6], $\lambda_{k}$ admits the following asymptotic expansion:

$$
\begin{aligned}
\lambda_{2}= & \frac{1}{n} \sum_{j=1}^{n}\left[\operatorname{cum}\left(m_{j}, m_{j}\right)+\operatorname{cum}^{2}\left(m_{j}\right)\right]=O(1), \\
\lambda_{3}= & \frac{1}{n} \sum_{j=1}^{n}\left[\operatorname{cum}\left(m_{j}, m_{j}, m_{j}\right)+3 \operatorname{cum}\left(m_{j}, m_{j}\right) \operatorname{cum}\left(m_{j}\right)+\operatorname{cum}^{3}\left(m_{j}\right)\right]=O(1), \\
\lambda_{4}= & \frac{1}{n} \sum_{j=1}^{n}\left[\operatorname{cum}\left(m_{j}, m_{j}, m_{j}, m_{j}\right)+4 \operatorname{cum}\left(m_{j}, m_{j}, m_{j}\right) \operatorname{cum}\left(m_{j}\right)\right. \\
& \left.+3 \operatorname{cum}^{2}\left(m_{j}, m_{j}\right)+6 \operatorname{cum}\left(m_{j}, m_{j}\right) \operatorname{cum}^{2}\left(m_{j}\right)+\operatorname{cum}^{4}\left(m_{j}\right)\right]=O(1) .
\end{aligned}
$$

The calculations for variance of higher moments (i.e. $\frac{1}{n} \sum_{j} m_{j}^{k}, k=2,3,4$ ) can be handled similarly as the proof of Lemma 3.1, albeit more tedious.

\section{Appendix C: Proofs of technical lemmas}

Proof of Lemma 3.2. Based on Lemmas B.2 and B.3, cumulants of $\sqrt{n} S R$ will be derived to establish its Edgeworth expansion. We only derive the first four cumulants because the higher order cumulants have smaller orders and can be neglected. From the definition of cumulants, we have

$$
\begin{aligned}
k_{1}= & \operatorname{cum}\left(\sqrt{n}\left(R_{1}+R_{2}+R_{3}\right)\right), \\
k_{2}= & \operatorname{cum}\left(n S R^{2}\right)-\operatorname{cum}^{2}\left(\sqrt{n}\left(R_{1}+R_{2}+R_{3}\right)\right), \\
k_{3}= & \operatorname{cum}\left(\sqrt{n} R_{1}, \sqrt{n} R_{1}, \sqrt{n} R_{1}\right) \\
& +3 \operatorname{cum}\left(\sqrt{n} R_{1}, \sqrt{n} R_{1}, \sqrt{n} R_{2}\right)+O\left(\frac{\log ^{3} n}{n^{3 / 2}}\right),
\end{aligned}
$$




$$
\begin{aligned}
k_{4}= & \operatorname{cum}\left(\sqrt{n} R_{1}, \sqrt{n} R_{1}, \sqrt{n} R_{1}, \sqrt{n} R_{1}\right) \\
& +4 \operatorname{cum}\left(\sqrt{n} R_{1}, \sqrt{n} R_{1}, \sqrt{n} R_{1}, \sqrt{n} R_{2}\right) \\
& +4 \operatorname{cum}\left(\sqrt{n} R_{1}, \sqrt{n} R_{1}, \sqrt{n} R_{1}, \sqrt{n} R_{3}\right) \\
& +6 \operatorname{cum}\left(\sqrt{n} R_{1}, \sqrt{n} R_{1}, \sqrt{n} R_{2}, \sqrt{n} R_{2}\right)+O\left(\frac{\log ^{4} n}{n^{2}}\right) .
\end{aligned}
$$

Consider $k_{1}$ in (C.1),

$$
\begin{aligned}
k_{1}= & \mathrm{E}\left(\sqrt{n}\left(R_{1}+R_{2}+R_{3}\right)\right) \\
= & \operatorname{cum}\left(\frac{\bar{m}}{\sqrt{\lambda_{2}}}\right)+\operatorname{cum}\left(\frac{1}{3} \frac{\lambda_{3} \bar{m}^{2}}{\lambda_{2}^{5 / 2}}-\frac{1}{2} \frac{\bar{m} \Delta_{2}}{\lambda_{2}^{3 / 2}}\right) \\
& +\operatorname{cum}\left(\frac{3}{8} \frac{\bar{m} \Delta_{2}^{2}}{\lambda_{2}^{5 / 2}}+\frac{1}{3} \frac{\bar{m}^{2} \Delta_{3}}{\lambda_{2}^{5 / 2}}-\frac{5}{6} \frac{\lambda_{3} \bar{m}^{2} \Delta_{2}}{\lambda_{2}^{7 / 2}}+\frac{4}{9} \frac{\lambda_{3}^{2} \bar{m}^{3}}{\lambda_{2}^{9 / 2}}-\frac{1}{4} \frac{\lambda_{4} \bar{m}^{3}}{\lambda_{2}^{7 / 2}}\right) .
\end{aligned}
$$

By Lemma 3.1, the bounds of terms in (C.5) can be directly derived, i.e.,

$$
\begin{aligned}
\operatorname{cum}(\bar{m}) & =O\left(\frac{\log ^{3} n}{n}\right)+O\left(n^{-1}\right), \\
\operatorname{cum}\left(\bar{m}^{2}\right) & =\operatorname{cum}\left(\frac{1}{n} \sum_{j=1}^{n} m_{j}, \frac{1}{n} \sum_{k=1}^{n} m_{k}\right)+\operatorname{cum}^{2}\left(\frac{1}{n} \sum_{j=1}^{n} m_{j}\right) \\
& =O\left(n^{-1}\right)+O\left(\frac{\log ^{3} n}{n}\right)^{2}, \\
\operatorname{cum}\left(\bar{m} \Delta_{2}\right) & =\operatorname{cum}\left(\frac{1}{n} \sum_{j=1}^{n} m_{j}, \frac{1}{n} \sum_{k=1}^{n} m_{k}^{2}\right)=O\left(n^{-1}\right) .
\end{aligned}
$$

In Gaussian case, the higher order cumulant $\operatorname{cum}\left(I_{n}\left(\omega_{j}\right) / f\left(\omega_{j}\right), I_{n}\left(\omega_{k}\right) / f\left(\omega_{k}\right)\right.$, $\left.I_{n}\left(\omega_{k}\right) / f\left(\omega_{k}\right)\right)$ can be decomposed into products of second order cumulants of normalized DFT, in which cumulants of order 3 and higher order vanish. To be specific, we have

$$
\begin{aligned}
& \operatorname{cum}\left(\frac{I_{n}\left(\omega_{j}\right)}{f\left(\omega_{j}\right)}, \frac{I_{n}\left(\omega_{k}\right)}{f\left(\omega_{k}\right)}, \frac{I_{n}\left(\omega_{k}\right)}{f\left(\omega_{k}\right)}\right) \\
= & 2 \operatorname{cum}^{2}\left(\frac{J_{n}\left(\omega_{j}\right)}{\sqrt{f\left(\omega_{j}\right)}}, \frac{J_{n}\left(\omega_{-k}\right)}{\sqrt{f\left(\omega_{-k}\right)}}\right) \operatorname{cum}\left(\frac{J_{n}\left(\omega_{k}\right)}{\sqrt{f\left(\omega_{k}\right)}}, \frac{J_{n}\left(\omega_{-k}\right)}{\sqrt{f\left(\omega_{-k}\right)}}\right) .
\end{aligned}
$$

Together with Lemma B.2, B.3, B.4 and B.5, the terms in (C.6) are of order

$$
\operatorname{cum}\left(\bar{m} \Delta_{2}^{2}\right)=\operatorname{cum}\left(\frac{1}{n} \sum_{i=1}^{n} m_{i}, \frac{1}{n} \sum_{j=1}^{n} m_{j}^{2}, \frac{1}{n} \sum_{k=1}^{n} m_{k}^{2}\right)
$$




$$
\begin{aligned}
& +\operatorname{cum}\left(\frac{1}{n} \sum_{j=1}^{n} m_{j}\right) \operatorname{cum}\left(\frac{1}{n} \sum_{j=1}^{n} m_{j}^{2}, \frac{1}{n} \sum_{k=1}^{n} m_{k}^{2}\right) \\
& =O\left(\frac{\log ^{3} n}{n^{2}}\right)+O\left(\frac{1}{n^{2}}\right),
\end{aligned}
$$

and

$$
\begin{aligned}
\operatorname{cum}\left(\bar{m}^{2} \Delta_{3}\right)= & \operatorname{cum}\left(\frac{1}{n} \sum_{i=1}^{n} m_{i}, \frac{1}{n} \sum_{j=1}^{n} m_{j}, \frac{1}{n} \sum_{k=1}^{n} m_{k}^{3}\right) \\
& +2 \operatorname{cum}\left(\frac{1}{n} \sum_{j=1}^{n} m_{j}\right) \operatorname{cum}\left(\frac{1}{n} \sum_{j=1}^{n} m_{j}, \frac{1}{n} \sum_{k=1}^{n} m_{k}^{3}\right) \\
= & O\left(\frac{\log ^{3} n}{n^{2}}\right)+O\left(\frac{1}{n^{2}}\right),
\end{aligned}
$$

and

$$
\begin{aligned}
\operatorname{cum}\left(\bar{m}^{2} \Delta_{2}\right)= & \operatorname{cum}\left(\frac{1}{n} \sum_{i=1}^{n} m_{i}, \frac{1}{n} \sum_{j=1}^{n} m_{j}, \frac{1}{n} \sum_{k=1}^{n} m_{k}^{2}\right) \\
& +2 \operatorname{cum}\left(\frac{1}{n} \sum_{j=1}^{n} m_{j}, \frac{1}{n} \sum_{k=1}^{n} m_{k}^{2}\right) \operatorname{cum}\left(\frac{1}{n} \sum_{j=1}^{n} m_{j}\right) \\
= & O\left(\frac{1}{n^{2}}\right)+O\left(\frac{\log ^{3} n}{n^{2}}\right),
\end{aligned}
$$

and

$$
\begin{aligned}
& \operatorname{cum}\left(\bar{m}^{3}\right) \\
= & \operatorname{cum}\left(\frac{1}{n} \sum_{i=1}^{n} m_{i}, \frac{1}{n} \sum_{j=1}^{n} m_{j}, \frac{1}{n} \sum_{k=1}^{n} m_{k}\right) \\
& +3 \operatorname{cum}\left(\frac{1}{n} \sum_{j=1}^{n} m_{j}, \frac{1}{n} \sum_{k=1}^{n} m_{k}\right) \operatorname{cum}\left(\frac{1}{n} \sum_{j=1}^{n} m_{j}\right)+\operatorname{cum}^{3}\left(\frac{1}{n} \sum_{j=1}^{n} m_{j}\right) \\
= & O\left(\frac{1}{n^{2}}\right)+O\left(\frac{\log ^{3} n}{n^{2}}\right)+O\left(\frac{\log ^{3} n}{n}\right)^{3} .
\end{aligned}
$$

This leads to the asymptotic expansion of $k_{1}$ in (3.4). The expansion of $k_{2}$ can be computed similarly. For $k_{3}$ and $k_{4}$, define

$$
\rho_{3}=\sum_{j=1}^{n}\left(\frac{\partial}{\partial \theta} \log f\left(\omega_{j}\right)\right)^{3} \operatorname{cum}\left(\frac{I_{n}\left(\omega_{j}\right)}{f\left(\omega_{j}\right)}, \frac{I_{n}\left(\omega_{j}\right)}{f\left(\omega_{j}\right)}, \frac{I_{n}\left(\omega_{j}\right)}{f\left(\omega_{j}\right)}\right),
$$




$$
\rho_{4}=\sum_{j=1}^{n}\left(\frac{\partial}{\partial \theta} \log f\left(\omega_{j}\right)\right)^{4} \operatorname{cum}\left(\frac{I_{n}\left(\omega_{j}\right)}{f\left(\omega_{j}\right)}, \frac{I_{n}\left(\omega_{j}\right)}{f\left(\omega_{j}\right)}, \frac{I_{n}\left(\omega_{j}\right)}{f\left(\omega_{j}\right)}, \frac{I_{n}\left(\omega_{j}\right)}{f\left(\omega_{j}\right)}\right) .
$$

Direct calculations give

$$
\begin{aligned}
\operatorname{cum}\left(\sqrt{n} R_{1}, \sqrt{n} R_{1}, \sqrt{n} R_{1}\right) & =\frac{2}{\lambda_{2}^{3 / 2}} \rho_{3}+o\left(n^{-1}\right), \\
\operatorname{cum}\left(\sqrt{n} R_{1}, \sqrt{n} R_{1}, \sqrt{n} R_{2}\right) & =-\frac{2}{3} \frac{1}{\lambda_{2}^{3 / 2}} \rho_{3}+o\left(n^{-1}\right) .
\end{aligned}
$$

Substituting the above into (C.3), we have

$$
k_{3,1}=\sqrt{n}\left\{\frac{2}{\lambda_{2}^{3 / 2}} \rho_{3}-3 \frac{2}{3} \frac{1}{\lambda_{2}^{3 / 2}} \rho_{3}\right\}=0,
$$

The calculations for the fourth order cumulants are similar. Again, direct calculations give

$$
\begin{aligned}
& \operatorname{cum}\left(\sqrt{n} R_{1}, \sqrt{n} R_{1}, \sqrt{n} R_{1}, \sqrt{n} R_{1}\right)=\frac{1}{n^{2}} \frac{\rho_{4}}{\lambda_{2}^{2}}+o\left(n^{-1}\right), \\
& \operatorname{cum}\left(\sqrt{n} R_{1}, \sqrt{n} R_{1}, \sqrt{n} R_{1}, \sqrt{n} R_{2}\right)=\frac{1}{2 n} \frac{\lambda_{3}^{2}}{\lambda_{2}^{3}}-\frac{3}{2 n^{2}} \frac{\rho_{4}}{\lambda_{2}^{2}}+O\left(\frac{\log ^{3} n}{n^{3}}\right), \\
& \operatorname{cum}\left(\sqrt{n} R_{1}, \sqrt{n} R_{1}, \sqrt{n} R_{1}, \sqrt{n} R_{3}\right)=-\frac{1}{12 n} \frac{\lambda_{3}^{2}}{\lambda_{2}^{3}}+\frac{1}{2 n^{2}} \frac{\rho_{4}}{\lambda_{2}^{2}}+o\left(n^{-1}\right)+O\left(\frac{\log ^{3} n}{n^{4}}\right), \\
& \operatorname{cum}\left(\sqrt{n} R_{1}, \sqrt{n} R_{1}, \sqrt{n} R_{2}, \sqrt{n} R_{2}\right)=-\frac{5}{18 n} \frac{\lambda_{3}^{2}}{\lambda_{2}^{3}}+\frac{1}{2 n^{2}} \frac{\rho_{4}}{\lambda_{2}^{2}}+o\left(n^{-1}\right)+O\left(\frac{\log ^{3} n}{n^{4}}\right) .
\end{aligned}
$$

Substituting the above into (C.4), we have

$$
k_{4,1}=\frac{1}{n} \frac{\rho_{4}}{\lambda_{2}^{2}}+4 \frac{1}{2} \frac{\lambda_{3}^{2}}{\lambda_{2}^{3}}-\frac{3}{2 n} \frac{\rho_{4}}{\lambda_{2}^{2}}-4 \frac{1}{12} \frac{\lambda_{3}^{2}}{\lambda_{2}^{3}}+\frac{1}{2 n} \frac{\rho_{4}}{\lambda_{2}^{2}}-6 \frac{5}{18} \frac{\lambda_{3}^{2}}{\lambda_{2}^{3}}+\frac{1}{2 n} \frac{\rho_{4}}{\lambda_{2}^{2}}=0 .
$$

In conclusion, we have $k_{3,1}=k_{4,1}=0$.

Proof of Lemma B.1. Since the function $\operatorname{cosec}(\mu)-\mu^{-1}$ is bounded on $\left(0, \frac{\pi}{2}\right)$, it follows that

$$
\begin{aligned}
& \int_{-\pi}^{\pi}\left|\frac{\sin (n \mu / 2)}{\sin (\mu / 2)}\right| d \mu \\
= & 4 \int_{0}^{\pi}\left|\frac{\sin (n \mu / 2)}{\mu}\right| d \mu+O(1) \\
= & 4\left(\int_{0}^{\pi} \frac{\sin \mu}{\mu} d \mu+\int_{0}^{\pi / n} \sin (n \mu / 2)\left\{\sum_{k=1}^{n-1} \frac{1}{\mu+k \pi / n}\right\} d \mu\right)+O(1) .
\end{aligned}
$$


The sum in the braces has lower- and upper-bound $\frac{n}{\pi} \sum_{j=1}^{n-1} \frac{1}{j}$ and $\frac{n}{\pi} \sum_{j=2}^{n} \frac{1}{j}$ respectively, thus equals to $\frac{n}{\pi}[\log n+O(1)]$. The proof is completed by noting that, from Brillinger [6],

$$
\int_{0}^{\pi / n} \sin (n \mu) d \mu=2 / n \quad \text { and } \quad \int_{0}^{\pi} \frac{\sin \mu}{\mu} d \mu=O(1) .
$$

Proof of Lemma B.2. Without loss of generality, assume that $\omega_{j} \leq \frac{\pi}{2}$ for sufficiently large $n$. Note that

$\mathrm{E}\left(I\left(\omega_{j}\right)-f\left(\omega_{j}\right)\right)=\mathrm{E}\left(J_{n}\left(\omega_{j}\right) J_{n}\left(\omega_{-j}\right)-f\left(\omega_{j}\right)\right)=\int_{-\pi}^{\pi} F_{n}\left(\omega_{j}-\mu\right)\left(f(\mu)-f\left(\omega_{j}\right)\right) d \mu$,

where $F_{n}(\mu)$ is the Fejér kernel $F_{n}(\mu)=\frac{1}{2 \pi n} \frac{\sin ^{2}(n \mu / 2)}{\sin ^{2}(\mu / 2)}$. Since $f(\mu)=\frac{1}{\mu^{2 d}} f^{*}(\mu)$, where $f^{*}(\mu)$ is a even function bounded above and bounded below from zero, we have

$$
\begin{aligned}
& \frac{1}{f\left(\omega_{j}\right)} \int_{-\pi}^{\pi} F_{n}\left(\omega_{j}-\mu\right)\left(f(\mu)-f\left(\omega_{j}\right)\right) d \mu \\
= & \frac{K}{2 \pi n} \int_{-\pi}^{\pi} \frac{\sin ^{2} n\left(\omega_{j}-\mu\right) / 2}{\sin ^{2}\left(\omega_{j}-\mu\right) / 2}\left(f(\mu)-f\left(\omega_{j}\right)\right) d \mu .
\end{aligned}
$$

The idea is to decompose the range $[-\pi, \pi]$ into five parts and establish a bound for each part,

$$
\frac{K}{n} \int_{-\pi}^{\pi}=\frac{K}{n}\left\{\int_{-\pi}^{\omega_{j}-\epsilon}+\int_{\omega_{j}-\epsilon}^{\omega_{j}-\frac{1}{n}}+\int_{\omega_{j}-\frac{1}{n}}^{\omega_{j}+\frac{1}{n}}+\int_{\omega_{j}+\frac{1}{n}}^{\omega_{j}+\epsilon}+\int_{\omega_{j}+\epsilon}^{\pi}\right\} .
$$

The first part of (C.7) is bounded by

$$
\begin{aligned}
\frac{K}{n} \int_{-\pi}^{\omega_{j}-\epsilon} & \leq \frac{K}{n}\left(\max _{\mu \in\left[-\pi, \omega_{j}-\epsilon\right]} \frac{\sin ^{2} n\left(\omega_{j}-\mu\right) / 2}{\sin ^{2}\left(\omega_{j}-\mu\right) / 2}\right) \int_{-\pi}^{\omega_{j}-\epsilon}\left|f(\mu)-f\left(\omega_{j}\right)\right| d \mu \\
& \leq \frac{K}{n} \int_{-\pi}^{\omega_{j}-\epsilon}\left(\left|\mu^{-2 d}\right|+\left|f\left(\omega_{j}\right)\right|\right) d \mu=O\left(n^{-1}\right) .
\end{aligned}
$$

The second part is bounded above by

$$
\begin{aligned}
\frac{K}{n} \int_{\omega_{j}-\epsilon}^{\omega_{j}-\frac{1}{n}} & \leq \frac{K}{n} \int_{\omega_{j}-\epsilon}^{\omega_{j}-\frac{1}{n}}\left(\frac{\sin ^{2} n\left(\omega_{j}-\mu\right) / 2}{\sin ^{2}\left(\omega_{j}-\mu\right) / 2} \max _{\mu} \frac{\partial}{\partial \mu} f(\mu)\left|\mu-\omega_{j}\right|\right) d \mu \\
& \leq \frac{K}{n} \int_{-\epsilon}^{-\frac{1}{n}} \frac{\sin ^{2}(n \lambda / 2)}{\sin ^{2}(\lambda / 2)}|\lambda| d \lambda \leq \frac{K}{n} \int_{-\epsilon}^{-\frac{1}{n}} \frac{1}{\lambda} d \lambda=O\left(\frac{\log n}{n}\right),
\end{aligned}
$$

where the third inequality follows from the Zygmund [39] that

$$
\frac{1}{n} \frac{\sin ^{2}(n \lambda / 2)}{\sin ^{2}(\lambda / 2)}=O\left(\left(n \lambda^{2}\right)^{-1}\right), \quad 0<|\lambda|<\pi
$$


The third part in (C.7) is bounded by

$$
\begin{aligned}
& \frac{K}{n}\left(\max _{\mu \in\left[\omega_{j}-\frac{1}{n}, \omega_{j}+\frac{1}{n}\right]} \frac{\partial}{\partial \mu} f(\mu)\right) \int_{\omega_{j}-\frac{1}{n}}^{\omega_{j}+\frac{1}{n}}\left(\frac{\sin ^{2} n\left(\omega_{j}-\mu\right) / 2}{\sin ^{2}\left(\omega_{j}-\mu\right) / 2}\right)\left|\mu-\omega_{j}\right| d \mu \\
\leq & \frac{K}{n} \int_{-\frac{1}{n}}^{\frac{1}{n}}\left(\frac{\sin ^{2} n \lambda / 2}{\sin ^{2} \lambda / 2}\right)|\lambda| d \lambda \leq \frac{K}{n^{2}} \int_{-\pi}^{\pi} \frac{\sin ^{2}(n \lambda / 2)}{\sin ^{2}(\lambda / 2)} d \lambda=O\left(n^{-1}\right) .
\end{aligned}
$$

The last step is obtained from

$$
\int_{-\pi}^{\pi}\left[\frac{\sin (n \lambda / 2)}{\sin (\lambda / 2)}\right]^{2} d \lambda=2 \pi n
$$

The fourth and fifth terms are bounded by $O(\log n / n)$ and $\frac{K}{n} \int_{\omega_{j}+\epsilon}^{\pi}=O\left(n^{-1}\right)$, respectively. The proof for $\mathrm{E}\left(J_{n}\left(\omega_{j}\right) J_{n}\left(\omega_{j}\right) / \sqrt{f\left(\omega_{j}\right) f\left(\omega_{j}\right)}\right)$ is similar.

Proof of Lemma B.3. Without loss of generality, assuming that $0<j \leq k<n$, we consider three situations

a. $j, k \in \Lambda_{1}$,

b. $j, k \in \Lambda_{2}$,

c. $j, k \in \Lambda_{3}$.

We prove that for each case, the covariances of normalized DFT at different Fourier frequencies are bounded by a term with order $\log n / n$. First, define

$$
F_{j, k}=\frac{1}{\sqrt{f\left(\omega_{j}\right) f\left(\omega_{-k}\right)}} \frac{1}{2 \pi n} \frac{\sin n\left(\omega_{j}-\mu\right) / 2}{\sin \left(\omega_{j}-\mu\right) / 2} \frac{\sin n\left(\omega_{k}-\mu\right) / 2}{\sin \left(\omega_{k}-\mu\right) / 2} .
$$

For case a, note that by using $\int_{-\pi}^{\pi} F_{j,-k}(\mu) d \mu=0$ for $j, k \in \Lambda_{1}$, we have

$$
\mathrm{E}\left(\frac{J_{n}\left(\omega_{j}\right) J_{n}\left(\omega_{k}\right)}{\sqrt{f\left(\omega_{j}\right) f\left(\omega_{k}\right)}}\right)=\int_{-\pi}^{\pi}\left[F_{j,-k}(\mu)\left(f(\mu)-f\left(\omega_{k}\right)\right)\right] d \mu
$$

The integral in (C.8) is divided into seven parts

$$
\int_{-\pi}^{\pi}=\int_{-\pi}^{-\omega_{k}-\epsilon}+\int_{-\omega_{k}-\epsilon}^{-\omega_{k}+\epsilon}+\int_{-\omega_{k}+\epsilon}^{-\frac{1}{n}}+\int_{-\frac{1}{n}}^{\frac{1}{n}}+\int_{\frac{1}{n}}^{\omega_{j}-\epsilon}+\int_{\omega_{j}-\epsilon}^{\omega_{j}+\epsilon}+\int_{\omega_{j}+\epsilon}^{\pi} .
$$

The terms $\int_{-\omega_{k}-\epsilon}^{-\omega_{k}+\epsilon}+\int_{\omega_{j}-\epsilon}^{\omega_{j}+\epsilon}$ in (C.9) are bounded by

$$
\begin{aligned}
& \frac{K}{n}\left\{\max _{\mu \in\left[-\omega_{k}-\epsilon,-\omega_{k}+\epsilon\right]}\left(\frac{1}{|2 \sin (\mu / 2)|^{2 d}} \frac{\left|\sin n\left(\omega_{j}-\mu\right) / 2\right|}{\left|\sin \left(2 \pi \omega_{j}-\mu\right) / 2\right|}\right)\left(\int_{-\omega_{k}-\epsilon}^{-\omega_{k}+\epsilon}+\int_{\omega_{j}-\epsilon}^{\omega_{j}+\epsilon}\right)\right. \\
& \left.+\max _{\mu \in\left[\omega_{j}-\epsilon, \omega_{j}+\epsilon\right]}\left(\frac{1}{|2 \sin (\mu / 2)|^{2 d}} \frac{\left|\sin n\left(\omega_{k}+\mu\right) / 2\right|}{\left|\sin \left(\omega_{k}+\mu\right) / 2\right|}\right)\left(\int_{-\omega_{k}-\epsilon}^{-\omega_{k}+\epsilon}+\int_{\omega_{j}-\epsilon}^{\omega_{j}+\epsilon}\right)\right\}
\end{aligned}
$$


$\leq \frac{K}{n} \int_{-\pi}^{\pi}\left|\frac{\sin (n \mu / 2)}{\sin (\mu / 2)}\right| d \mu=O\left(\frac{\log n}{n}\right)$,

where the last step follows from Lemma B.1. Also, $\int_{-\pi}^{-\omega_{k}-\epsilon}+\int_{\omega_{j}+\epsilon}^{\pi}$ are bounded by $O\left(n^{-1}\right)$. Finally,

$$
\begin{aligned}
& \frac{K}{2 \pi n}\left(\int_{-\frac{1}{n}}^{\frac{1}{n}}+\int_{\frac{1}{n}}^{\omega_{j}-\epsilon}+\int_{-\omega_{k}+\epsilon}^{-\frac{1}{n}}\right) \\
\leq & \frac{K}{n} \int_{-\frac{1}{n}}^{\frac{1}{n}}\left(\left|\mu^{-2 d}\right|+\left|\omega_{j}\right|^{-2 d}\right) d \mu \\
& +\left(\max _{\mu \in\left[\frac{1}{n}, \omega_{j}-\epsilon\right]} \frac{\sin n\left(\omega_{j}-\mu\right) / 2}{\sin \left(\omega_{j}-\mu\right) / 2}\right) \int_{\frac{1}{n}}^{\omega_{j}-\epsilon}\left(\left|\mu^{-2 d}\right|+\left|\omega_{j}\right|^{-2 d}\right) d \mu \\
& +\left(\max _{\mu \in\left[-\omega_{k}+\epsilon,-\frac{1}{n}\right]} \frac{\sin n\left(\omega_{k}+\mu\right) / 2}{\sin \left(\omega_{k}+\mu\right) / 2}\right) \int_{-\omega_{k}+\epsilon}^{-\frac{1}{n}}\left(\left|\mu^{-2 d}\right|+\left|\omega_{j}\right|^{-2 d}\right) d \mu \\
= & O\left(n^{-1}\right) .
\end{aligned}
$$

Next, to prove $\mathrm{E}\left(J_{n}\left(\omega_{j}\right) J_{n}\left(\omega_{-k}\right) / \sqrt{f\left(\omega_{j}\right) f\left(\omega_{-k}\right)}\right)=O(\log n / n)$ for a, note that

$$
\begin{aligned}
\mathrm{E}\left(\frac{J_{n}\left(\omega_{j}\right) J_{n}\left(\omega_{-k}\right)}{\left.\sqrt{f\left(\omega_{j}\right) f\left(\omega_{-k}\right)}\right)=}\right. & \int_{\left(\omega_{j}+\omega_{k}\right) / 2}^{2 \omega_{j}}\left(f(\mu)-f\left(\omega_{j}\right)\right) F_{j, k}(\mu) d \mu \\
& +\int_{\omega_{k} / 2}^{\left(\omega_{j}+\omega_{k}\right) / 2}\left(f(\mu)-f\left(\omega_{k}\right)\right) F_{j, k}(\mu) d \mu \\
& -\left(f\left(\omega_{j}\right)-f\left(\omega_{k}\right)\right) \int_{\omega_{k} / 2}^{\left(\omega_{j}+\omega_{k}\right) / 2} F_{j, k}(\mu) d \mu \\
& +\left(\int_{2 \omega_{j}}^{\pi}+\int_{-\pi}^{\omega_{k} / 2}\right)\left(f(\mu)-f\left(\omega_{j}\right)\right) F_{j, k}(\mu) d \mu .
\end{aligned}
$$

For the first part, since

$$
\left|\frac{\sin n\left(\omega_{j}-\mu\right) / 2}{\sin \left(\omega_{j}-\mu\right) / 2}\right| \leq\left|\frac{2}{\omega_{j}-\mu}\right|, \quad 0<\left|\omega_{j}-\mu\right|<\pi,
$$

it follows that

$$
\begin{aligned}
& \int_{\left(\omega_{j}+\omega_{k}\right) / 2}^{2 \omega_{j}}\left(f(\mu)-f\left(\omega_{j}\right)\right) F_{j, k}(\mu) d \mu \\
\leq & \frac{K}{2 \pi n}\left(\max _{\left(\omega_{j}+\omega_{k}\right) / 2 \leq \mu \leq 2 \omega_{j}} \frac{\partial}{\partial \mu} f(\mu)\right) \int_{\left(\omega_{j}+\omega_{k}\right) / 2}^{2 \omega_{j}}\left|\frac{\sin n\left(\omega_{k}-\mu\right) / 2}{\sin \left(\omega_{k}-\mu\right) / 2}\right| d \mu \\
\leq & \frac{K}{2 \pi n} \int_{-\pi}^{\pi}\left|\frac{\sin (n \lambda / 2)}{\sin (\lambda / 2)}\right| d \lambda=O\left(\frac{\log n}{n}\right) .
\end{aligned}
$$


For the second part, we have

$$
\int_{\omega_{k} / 2}^{\left(\omega_{j}+\omega_{k}\right) / 2}\left(f(\mu)-f\left(\omega_{k}\right)\right) F_{j, k}(\mu) d \mu=O\left(\frac{\log n}{n}\right) .
$$

The third part is bounded by

$$
\begin{aligned}
& \left(\omega_{j}-\omega_{k}\right)\left(\max _{\omega_{k} \leq \mu \leq \omega_{j}} \frac{\partial}{\partial \mu} f(\mu)\right) \frac{K}{2 \pi n} \int_{\omega_{k} / 2}^{\left(\omega_{j}+\omega_{k}\right) / 2}\left(\frac{2}{\left|\omega_{j}-\mu\right|} \frac{\sin n\left(\omega_{k}-\mu\right) / 2}{\sin \left(\omega_{k}-\mu\right) / 2}\right) d \mu \\
\leq & \left(\omega_{j}-\omega_{k}\right)\left(\max _{\omega_{k} / 2 \leq \mu \leq\left(\omega_{j}+\omega_{k}\right) / 2} \frac{1}{\left|\omega_{j}-\mu\right|}\right) \frac{K}{2 \pi n} \int_{\omega_{k} / 2}^{\left(\omega_{j}+\omega_{k}\right) / 2}\left|\frac{\sin n\left(\omega_{k}-\mu\right) / 2}{\sin \left(\omega_{k}-\mu\right) / 2}\right| d \mu \\
= & O\left(\frac{\log n}{n}\right) .
\end{aligned}
$$

For the last part, we have

$$
\begin{aligned}
& \int_{-\pi}^{\omega_{k} / 2}\left(f(\mu)-f\left(\omega_{j}\right)\right) F_{j, k}(\mu) d \mu \\
\leq & \frac{K}{2 \pi n}\left(\max _{-\epsilon \leq \mu \leq \epsilon} \frac{\sin n\left(\omega_{j}-\mu\right) / 2}{\sin \left(\omega_{j}-\mu\right) / 2} \frac{\sin n\left(\omega_{k}-\mu\right) / 2}{\sin \left(\omega_{k}-\mu\right) / 2}\right) \int_{-\epsilon}^{\epsilon}\left(|\mu|^{-2 d}+\left|\omega_{j}\right|^{-2 d}\right) d \mu \\
= & O\left(n^{-1}\right)
\end{aligned}
$$

and

$$
\int_{2 \omega_{j}}^{\pi}\left(f(\mu)-f\left(\omega_{j}\right)\right) F_{j, k}(\mu) d \mu=O\left(n^{-1}\right) .
$$

Thus, for a, we have

$$
\mathrm{E}\left(\frac{J_{n}\left(\omega_{j}\right) J_{n}\left(\omega_{k}\right)}{\sqrt{f\left(\omega_{j}\right) f\left(\omega_{k}\right)}}\right)=O\left(\frac{\log n}{n}\right) \text { and } \mathrm{E}\left(\frac{J_{n}\left(\omega_{j}\right) J_{n}\left(\omega_{-k}\right)}{\sqrt{f\left(\omega_{j}\right) f\left(\omega_{-k}\right)}}\right)=O\left(\frac{\log n}{n}\right) .
$$

For the argument for b is similar to the proof of a, so we omit the details. For c, note that

$$
\begin{aligned}
\mathrm{E}\left(\frac{J_{n}\left(\omega_{j}\right) J_{n}\left(\omega_{k}\right)}{\sqrt{f\left(\omega_{j}\right) f\left(\omega_{k}\right)}}\right) & \leq K \omega_{k}^{2 d} \int_{-\pi}^{\pi} \frac{F_{j k}(\mu)}{|2 \sin (\mu / 2)|^{2 d}} d \mu \\
& \leq \frac{K}{2 \pi n} \frac{|2 \pi k|^{2 d}}{n^{2 d}}\left[\int_{-\pi}^{-\epsilon}+\int_{-\epsilon}^{\epsilon}+\int_{\epsilon}^{\omega_{j}-\epsilon}+\int_{\omega_{j}-\epsilon}^{\omega_{j}+\epsilon}+\int_{\omega_{j}+\epsilon}^{\pi}\right]
\end{aligned}
$$

The second term is bounded by

$$
\begin{aligned}
& \frac{K}{2 \pi n} \frac{|2 \pi k|^{2 d}}{n^{2 d}} \max _{\mu \in[-\epsilon, \epsilon]}\left(\frac{\sin n\left(\omega_{j}-\mu\right) / 2}{\sin \left(\omega_{j}-\mu\right) / 2}\right) \int_{-\pi}^{\pi}\left|\frac{\sin n\left(\frac{2 \pi k}{n}+\mu\right) / 2}{\sin \left(\frac{2 \pi k}{n}+\mu\right) / 2} \frac{1}{|2 \sin (\mu / 2)|^{2 d}}\right| d \mu \\
& \quad=O\left(n^{-1}\right) .
\end{aligned}
$$

The calculations for other terms are similar and bounds of the terms are of order $\log n / n$. It is also true that $\mathrm{E}\left(J_{n}\left(\omega_{j}\right) J_{n}\left(\omega_{-k}\right) / \sqrt{f\left(\omega_{j}\right) f\left(\omega_{-k}\right)}\right)=O(\log n / n)$. Combining a-c, Lemma B.3 follows. 
Proof of Lemma B.6. Since

$$
\mathrm{E}\left(J_{n}\left(\omega_{j}\right) J_{n}\left(\omega_{-j}\right)-f\left(\omega_{j}\right)\right)=\int_{-\pi}^{\pi} F_{n}\left(\omega-\omega_{j}\right)\left(f(\omega)-f\left(\omega_{j}\right)\right) d \omega,
$$

where $F_{n}(\omega)$ is the Fejér kernel, it suffices to show that

$$
\int_{-\pi}^{\pi} F_{n}\left(\omega-\omega_{j}\right)\left(f(\omega)-f\left(\omega_{j}\right)\right) d \omega \geq \frac{K}{j} \omega_{j}^{-2 d} .
$$

Decompose the integral on the left hand side of (C.11) into five parts

$$
\int_{-\pi}^{\pi}=\int_{\epsilon}^{\pi}+\int_{2 \omega_{j}}^{\epsilon}+\int_{-2 \omega_{j}}^{2 \omega_{j}}+\int_{-\epsilon}^{-2 \omega_{j}}+\int_{-\pi}^{-\epsilon}
$$

where for sufficiently large $n, 2 \omega_{j}<\epsilon<\pi / 2$. The first term of (C.12) is bounded below by

$$
\begin{aligned}
\int_{\epsilon}^{\pi} & \geq \min _{\epsilon \leq \omega \leq \pi}\left\{f(\omega)-f\left(\omega_{j}\right)\right\} \int_{\epsilon}^{\pi} \frac{1}{2 \pi n} \frac{\sin ^{2} n\left(\omega-\omega_{j}\right) / 2}{\sin ^{2}\left(\omega-\omega_{j}\right) / 2} d \omega \\
& \geq \frac{K}{2 \pi n} \int_{\epsilon-\omega_{j}}^{\pi-\omega_{j}} \frac{\sin ^{2} n \omega / 2}{\sin ^{2} \omega / 2} d \omega=\frac{K}{n} .
\end{aligned}
$$

For small $\epsilon$, let $\delta_{\epsilon}=\left[\frac{\epsilon-2 \omega_{j}}{n}\right]$, then using the property of Fejér kernel [33], the second term of (C.12) is bounded below by

$$
\begin{aligned}
\left|\int_{2 \omega_{j}}^{\epsilon}\right| & \geq \min _{2 \omega_{j} \leq \omega \leq \epsilon}\left(\frac{\partial}{\partial \omega} f(\omega)\right) \int_{2 \omega_{j}}^{\epsilon} \frac{1}{2 \pi n} \frac{\sin ^{2} n\left(\omega-\omega_{j}\right) / 2}{\sin ^{2}\left(\omega-\omega_{j}\right) / 2}\left(\omega-\omega_{j}\right) d \omega \\
& \geq \frac{K \epsilon^{-1-2 d}}{n} \sum_{k=0}^{n-1} \int_{\omega_{j}+\delta_{\epsilon} k}^{\omega_{j}+\delta_{\epsilon}(k+1)} \frac{\sin ^{2} n \omega / 2}{\sin ^{2} \omega / 2} \omega d \omega \\
& \geq \frac{K}{n} \sum_{k=0}^{n-1}\left(\min _{\omega_{j}+\delta_{\epsilon} k \leq \omega \leq \omega_{j}+\delta_{\epsilon}(k+1)} \omega\right) \int_{\omega_{j}+\delta_{\epsilon} k}^{\omega_{j}+\delta_{\epsilon}(k+1)} \frac{\sin ^{2} n \omega / 2}{\sin ^{2} \omega / 2} d \omega \\
& \geq \sum_{k=0}^{n-1} \frac{K}{n^{2}}\left(\omega_{j}+\delta_{\epsilon} k\right)=K\left\{\frac{\omega_{j}}{n}+\frac{\epsilon-2 \omega_{j}}{n}\right\} \geq \frac{K}{n} .
\end{aligned}
$$

By an identical argument, we have $\int_{-\pi}^{-\epsilon} \geq K / n$ and $\int_{-\epsilon}^{-2 \omega_{j}} \geq K / n$. For $\int_{-2 \omega_{j}}^{2 \omega_{j}}$ in (C.12), we have

$$
\int_{-2 \omega_{j}}^{2 \omega_{j}}=\int_{-2 \omega_{j}}^{\omega_{j}-2 \pi / n}+\int_{\omega_{j}-2 \pi / n}^{\omega_{j}+2 \pi / n}+\int_{\omega_{j}+2 \pi / n}^{2 \omega_{j}}=L_{1}+L_{2}+L_{3}
$$

Note that $L_{3}$ is bounded below by

$$
\min _{\omega_{j}+2 \pi / n \leq \omega \leq 2 \omega_{j}}\left(\frac{\partial}{\partial \omega} f(\omega)\right) \int_{\omega_{j}+2 \pi / n}^{2 \omega_{j}} \frac{1}{2 \pi n} \frac{\sin ^{2} n\left(\omega-\omega_{j}\right) / 2}{\sin ^{2}\left(\omega-\omega_{j}\right) / 2}\left(\omega-\omega_{j}\right) d \omega
$$




$$
\begin{aligned}
& \geq K \omega_{j}^{-(1+2 d)} \int_{2 \pi / n}^{\omega_{j}} \frac{1}{2 \pi n} \frac{\sin ^{2} n \omega / 2}{\sin ^{2} \omega / 2} \omega d \omega \\
& \geq K \omega_{j}^{-(1+2 d)}\left(\min _{2 \pi / n \leq \omega \leq \omega_{j}} \omega\right) \int_{2 \pi / n}^{\omega_{j}} \frac{1}{2 \pi n} \frac{\sin ^{2} n \omega / 2}{\sin ^{2} \omega / 2} d \omega \\
& =K \omega_{j}^{-(1+2 d)} \frac{1}{n} \int_{2 \pi / n}^{\omega_{j}} \frac{1}{2 \pi n} \frac{\sin ^{2} n \omega / 2}{\sin ^{2} \omega / 2} d \omega .
\end{aligned}
$$

Since the integral in the last equation is bounded above by small $\epsilon>0$ from Section 6.1 in Priestley [33], lower bound of $L_{3}$ is no more than $K \frac{1}{j} \omega_{j}^{-2 d}$. By symmetry, it is also true that lower bound of $L_{1}$ is no more than $K \frac{1}{j} \omega_{j}^{-2 d}$. Next,

$$
\begin{aligned}
L_{2} & =\left(\int_{-2 \pi / n}^{-1 / n}+\int_{-1 / n}^{1 / n}+\int_{1 / n}^{2 \pi / n}\right) \frac{1}{2 \pi n} \frac{\sin ^{2} n \omega / 2}{\sin ^{2} \omega / 2}\left\{\left(\omega+\omega_{j}\right)^{-2 d}-\omega_{j}^{-2 d}\right\} d \omega \\
& =L_{21}+L_{22}+L_{23} .
\end{aligned}
$$

Note that

$$
L_{22} \geq \omega_{j}^{-1-2 d} \int_{-1 / n}^{1 / n} \frac{1}{2 \pi n} \frac{\sin ^{2} n \omega / 2}{\sin ^{2} \omega / 2} \omega d \omega=0
$$

For $L_{23}$,

$$
L_{23} \geq\left\{\min _{\omega \in[1 / n, 2 \pi / n]} \min _{\xi \in\left[\omega_{j}, \omega_{j}+\omega\right]} \xi^{-1-2 d} \omega\right\} \int_{1 / n}^{2 \pi / n} \frac{1}{2 \pi n} \frac{\sin ^{2} n \omega / 2}{\sin ^{2} \omega / 2} d \omega \geq K \frac{1}{j} \omega_{j}^{-2 d} .
$$

We also have $L_{21} \geq K \frac{1}{j} \omega_{j}^{-2 d}$. From the above analysis, the division $\int_{-2 \omega_{j}}^{2 \omega_{j}}$ is bounded below by $K \frac{1}{j} \omega_{j}^{-2 d}$. Collecting the lower bounds of each part in (C.12), (B.3) is established.

\section{References}

[1] Andrews, D.W.K. And Liberman, O. (2005). Valid Edgeworth expansions for the whittle maximum likelihood estimator for stationary longmemory gaussian time series. Econometric Theory 21, 710-734. MR2189493

[2] An, H.Z., Chen, Z.G. And Hannan, E.J. (1983). The maximum of the periodogram. Journal of Multivariate Analysis 13, 383-400. MR0716931

[3] Barndorff-Nielsen, O.E. And Hall, P.(1988). On the level-error after Bartlett adjustment of the likelihood ratio statistic. Biometrika 75, 374-378. MR0946056

[4] Beran, J. (1994) Statistics for Long-Memory Processes. Chapman \& Hall, New York. MR1304490

[5] Bhattacharya, R.N. and Ghosh, J.K. (1974). On the validity of the formal Edgeworth expansion. Annals of Statistics 6, 434-451. MR0471142

[6] Brillinger, D.R. (1981). Time Series: Data Analysis and Theory. HoldenDay, San Francisco. MR0595684 
[7] Chambers, J.M. (1967). On methods of asymptotic approximation for multivariate distributions. Biometrika 54, 367-383. MR0225429

[8] Chan, N.H. AND Ling, S. (2006). Empirical likelihood for GARCH models. Econometric Theory 22, 402-428. MR2256098

[9] Chan, N.H. AND LiU, L. (2010). Bartlett correctability of empirical likelihood in time series. Journal of The Japanese Statistical Society 40, $1-5$.

[10] Chen, X.S. And Cui, H. (2007). On the second order properties of empirical likelihood with moment restrictions. Journal of Econometrics 141, 492-516. MR2413478

[11] Chen, X.S. and Hall, P. (1993). Smoothed empirical likelihood confidence intervals for quantiles. Annals of Statistics 21, 1166-1181. MR1241263

[12] Chuang, C.S. and Chan, N.H. (2002). Empirical likelihood for autoregressive models with applications to unstable time series. Statistica Sinica 12, 387-407. MR1902716

[13] Dahlhaus, R. (1989). Efficient parameter estimation for self-similar processes. Annals of Statistics 17, 1749-1766. MR1026311

[14] Dahlhaus, R. And Janas D. (1996). A frequency domain bootstrap for ratio statistics in time series analysis. Annals of Statistics 24, 1934-1963. MR1421155

[15] DiCiccio, T.J., Hall, P. and Romano, J.P. (1991). Empirical likelihood is Bartlett correctable. Annals of Statistics 19, 1053-1061. MR1105861

[16] DiCiccio, T.J. And Romano, J.P. (1989). On adjustment based on the signed root of the empirical likelihood ratio statistics. Biometrika $\mathbf{7 6}, 447-$ 456. MR1040639

[17] Granger, C.W.J. And Joyeux, R. (1980). An introduction to longmemory time series and fractional differencing. Journal of Time Series Analysis 1, 15-30. MR0605572

[18] Hall, P. (1992). The Bootstrap and Edgeworth Expansion. Springer, New York. MR1145237

[19] Hall, P. And La Scala, B. (1990). Methodology and algorithms of empirical likelihood. Internat. Statist. Rev. 58, 109-127.

[20] Hosking, J.R.M. (1981). Fractional differencing. Biometrika 68, 165-176. MR0614953

[21] Hosoya, Y. (1997). A limit theory for long-range dependence and statistical inference on related models. Annals of Statistics 25, 105-137. MR1429919

[22] Hurvich, C.M. and Beltrao K.I. (1993). Asymptotics for the lowfrequency ordinates of the periodogram of a long-memory time series. Journal of Time Series Analysis 14, 455-472. MR1243575

[23] Hurvich, C.M. And Zeger, S. (1987). Frequency domain bootstrap methods for time series. Statistics and Operations Research Working Paper, New York University, New York.

[24] Kitamura, Y. (1997). Empirical likelihood methods with weakly dependent processes. Annals of Statistics 25, 2084-2102. MR1474084 
[25] Kreiss, J.-P. And Paparoditis, E. (2011). Bootstrap for dependent data: a review (with discussion). Journal of the Korean Statistical Society 40, 357-395. MR2906623

[26] Mandelbrot, B.B. And Van Ness, J.W. (1968). Fractional Brownian motions, fractional noises and applications. SIAM Review 10, 422-437. MR0242239

[27] Monti, A.C. (1997). Empirical likelihood confidence regions in time series models. Biometrika 84, 395-405. MR1467055

[28] Nordman, D.J. AND LahiRI, S.N. (2006). A frequency domain empirical likelihood for short- and long-range dependence. Annals of Statistics 34, 3019-3050. MR2329476

[29] OGata, H. (2005). Empirical likelihood approach for non-Gaussian stationary processes. Scientiae Mathematicae Japonicae 62, 429-438. MR2182617

[30] Owen, A.B. (1988). Empirical likelihood ratio confidence intervals for a single functional. Biometrika 75, 237-249. MR0946049

[31] Owen, A.B. (1990). Empirical likelihood ratio confidence regions. Annals of Statistics 18, 90-120. MR1041387

[32] Owen, A.B. (2001). Empirical Likelihood. Chapman \& Hall, New York.

[33] Priestley, M.B. (1981). Spectral Analysis and Time Series. Academic Press.

[34] QIn, J. AND Lawless, J. (1994). Empirical likelihood and general estimating functions. Annals of Statistics 22, 300-325. MR1272085

[35] Robinson, P.M. (1995). Log-periodogram regression of time series with long range dependence. Annals of Statistics 23, 1048-1072. MR1345214

[36] Whittle, P. (1953). Estimation and information in stationary time series. Archiv. Math. 2, 423-434. MR0060797

[37] YAU, C.Y. (2012). Empirical likelihood in long-memory time series models. Journal of Time Series Analysis 33, 269-275. MR2902463

[38] Zhang, B. (1996). On the accuracy of empirical likelihood confidence intervals for M-Functionals. Journal of Nonparametric Statistics 6, 311-321. MR1386342

[39] Zygmund, A. (1977). Trigonometric Series. Cambridge Univ. Press. MR0617944 